\title{
Lenalidomide in multiple myeloma: an evidence-based review of its role in therapy
}

This article was published in the following Dove Press journal:

Core Evidence

6 November 2009

Number of times this article has been viewed

\section{Paul Richardson \\ Constantine Mitsiades \\ Jacob Laubach \\ Robert Schlossman \\ Irene Ghobrial \\ Teru Hideshima \\ Nikhil Munshi \\ Kenneth Anderson}

Jerome Lipper Multiple Myeloma Center, Division of Hematologic Malignancies, Dana-Farber Cancer Institute, Harvard Medical School, Boston, MA, USA
Correspondence: Paul Richardson

Dana-Farber Cancer Institute, Harvard Medical School, 44 Binney Street, Dana IB02, Boston, MA 02II5, USA

$\mathrm{Tel}+\mid 6176322127$ or $+\mid 6176322104$ Fax + I 67I 632430 I or $+\mid 617632430$ I Email paul_richardson@dfci.harvard.edu

Introduction: Multiple myeloma (MM) is a relatively common and incurable hematological malignancy. Currently, there is no single standard therapy, with choice of treatment dependent on individual patient factors. Lenalidomide is an immunomodulatory drug with potent antitumor, antiangiogenic, immunomodulatory, and proapoptotic activity in MM.

Aims: To evaluate the evidence for the use of lenalidomide in its current indication in relapsed or refractory MM, and additionally its investigational use for the treatment of newly diagnosed MM.

Evidence review: In patients with relapsed and refractory MM, adding lenalidomide to high-dose dexamethasone significantly improves response rates and time-to-progression, relative to high-dose dexamethasone alone. This translates into a significant extension of overall survival (with a median extension of 9.1 months in a pivotal phase III study). Outcome is independent of patient age, number of previous therapies, type of previous therapy (including thalidomide or autologous stem cell transplantation), renal impairment, and $\beta_{2}$-microglobulin level. Evidence suggests that combining lenalidomide with low-dose dexamethasone improves outcomes in patients with newly diagnosed disease and is superior to lenalidomide combined with highdose dexamethasone. Myelosuppression is the predominant toxicity observed, although some studies have shown high incidences of venous thromboembolism in the absence of prophylactic antithrombotic anticoagulation therapy. There is currently only limited evidence regarding the health economics of lenalidomide.

Role in therapy: The encouraging results obtained with lenalidomide alone and in combination with dexamethasone in patients with relapsed or refractory MM have led to its adoption as a recommended therapy in patients who have received at least one prior treatment. Emerging evidence supports the ongoing investigation of lenalidomide in combination with low-dose dexamethasone, and in other combinations including bortezomib, for use both in relapsed, refractory, and newly diagnosed MM.

Keywords: lenalidomide, evidence, multiple myeloma, outcomes, treatment

Core evidence clinical impact summary for lenalidomide in relapsed, refractory multiple myeloma

\begin{tabular}{|lll|}
\hline Outcome measure & Evidence & Implications \\
\hline $\begin{array}{l}\text { Patient-oriented evidence } \\
\text { Prolongation of OS }\end{array}$ & Substantial & $\begin{array}{l}\text { Median OS is significantly longer with } \\
\text { lenalidomide plus dexamethasone than with } \\
\text { dexamethasone alone }\end{array}$ \\
Prolongation of TTP & Substantial & $\begin{array}{l}\text { Median TTP is significantly longer with } \\
\text { lenalidomide plus dexamethasone than with } \\
\text { dexamethasone alone }\end{array}$ \\
& &
\end{tabular}




\begin{tabular}{|lll|}
\hline (Continued) & & \\
\hline Outcome measure & Evidence & Implications \\
\hline Adverse events & Substantial & $\begin{array}{l}\text { A significantly higher proportion of patients } \\
\text { treated with lenalidomide plus dexametha- } \\
\text { sone report grade } 3 \text { or } 4 \text { adverse events, } \\
\text { with neutropenia, thrombocytopenia, anemia, } \\
\text { and venous thromboembolic events being the } \\
\text { most important }\end{array}$ \\
$\begin{array}{l}\text { Improvement in quality } \\
\text { of life }\end{array}$ & No evidence & \\
$\begin{array}{l}\text { Disease-oriented evidence } \\
\text { Improvement in response } \\
\text { rate (overall and } \\
\text { complete response) }\end{array}$ & Substantial & $\begin{array}{l}\text { A significantly higher proportion of patients } \\
\text { treated with lenalidomide plus dexametha- } \\
\text { sone compared with dexamethasone alone } \\
\text { respond to treatment }\end{array}$ \\
$\begin{array}{l}\text { Economic evidence } \\
\text { Health care resource } \\
\text { utilization }\end{array}$ & Limited & $\begin{array}{l}\text { Direct drug costs of the approved lenalido- } \\
\text { mide regimen appear to be similar to or } \\
\text { higher than related novel agent regimens }\end{array}$ \\
\hline $\begin{array}{l}\text { Cost effectiveness } \\
\text { Abbreviations: OS, overall survival;TTP, time to progression. }\end{array}$
\end{tabular}

\section{Scope, aims, and objectives}

Lenalidomide $^{1}$ in combination with dexamethasone is indicated for the treatment of multiple myeloma (MM) in patients who have received at least one prior therapy. ${ }^{2,3}$ This review provides a background to $\mathrm{MM}$, summarizes current therapies and unmet needs, and evaluates the current evidence for the use of lenalidomide. Disease-oriented outcomes are evaluated, including response rates, response duration, time-to-progression (TTP), overall survival (OS), and one-year survival, as well as safety and tolerability. A search of the literature to-date did not identify any studies with patient-reported outcomes, such as quality of life, functional status, treatment satisfaction, adherence, or symptom relief. These parameters of clinical benefit are therefore not included in this review.

\section{Methods}

The English language medical literature was reviewed to identify abstracts and articles relating to lenalidomide in MM. Relevant databases were searched on April 11th, 2008 using the search terms "lenalidomide OR Revlimid OR CC-5013 AND 'multiple myeloma". Each database was searched from the beginning of the database to the date of the search, unless otherwise specified. The following databases, including proceedings of oncology-based meetings, were searched for relevant abstracts and full text articles:

- PubMed, http://www.ncbi.nlm.nih.gov/entrez

- Cochrane Database of Systematic Reviews (CDSR), http://www.cochrane.org
- ClinicalTrials.gov clinical trial register, http://www. clinicaltrials.gov

- ClinicalStudyResults.org clinical trial register, http:// www.clinicalstudyresults.org

- American Society of Hematology (ASH), 2005-2007, http://bloodjournal.hematologylibrary.org/search.dtl

- International Myeloma Working Group (IMWG), 2007

- European Hematology Association (EHA), 2008, http:// www.ehaweb.org/

- American Society of Clinical Oncology (ASCO), 2005-2007, http://www.asco.org/portal/site/ASCO

An additional search of the English language literature on PubMed conducted on April 11, 2008 was further refined to provide specificity for the search using the limits "clinical trial", "meta analysis", "randomized controlled trial", and "humans". The original PubMed search yielded 228 records including abstracts, and was subsequently narrowed to 18 records with these limits imposed. Six records were excluded from these 18 records for reasons of relevance $(\mathrm{n}=2)$, incorrect indication $(\mathrm{n}=3)$, and nonsystematic review $(\mathrm{n}=1)$. A further search of PubMed conducted on September 1, 2008 and time-limited from the previous search but otherwise using the same limits yielded one additional record, which was included. A similar search of the ASCO website on the same day yielded 13 additional abstracts, while handsearching of the European Hematology Association (EHA; http://www.ehaweb.org/) 2008 meeting abstracts produced 10 new abstracts. Eighteen of these records were included in the clinical evidence. No systematic reviews were identified 
for the use of lenalidomide in MM. Two papers and 18 abstracts were of level 2 evidence, and another 11 papers and 25 abstracts were of level $\geq 3$ evidence. The levels of evidence identified from the literature searches are summarized in Table 1. Criteria for exclusion were nonsystematic reviews, case studies, case series, phase I clinical trials or interim analyses of phase I/II clinical trials, and duplicate abstracts defined as presentation of similar data in the same calendar year. Substudy analyses were included at the same level of evidence as for the original study. Descriptive and observational studies, including retrospective studies, were included only for evaluation of safety.

\section{Disease overview}

$\mathrm{MM}$ is a hematological malignancy of plasma cells characterized by clonal expansion, bone marrow infiltration, lytic bone disease, hypercalcemia, renal insufficiency, and the presence, in the vast majority of patients, of immunoglobulin paraproteins (M-protein) in the serum and/or urine. ${ }^{4}$ The disease arises from a B-cell of the normal germinal center as a result of a chromosomal translocation that places an oncogene under the control of immunoglobulin enhancers. ${ }^{5}$ Despite recent therapeutic advances, including high-dose chemotherapy and autologous stem cell transplantation (SCT), MM is an incurable disease with a median overall survival (OS) of three to four years and a five-year relative survival of approximately $33 \%$ in $2007 .{ }^{6,7}$ During the past 10 years, survival rates for MM have increased; however, relapse remains inevitable and, until recently, there were few effective salvage therapies. ${ }^{8}$

Table I Evidence base included in the review

\begin{tabular}{lll}
\hline Category & \multicolumn{2}{l}{ Number of records } \\
\cline { 2 - 3 } & Full papers & Abstracts \\
\hline Initial search & 228 & 216 \\
Records excluded & 216 & 191 \\
Records included & 12 & 25 \\
Search update, new records & 1 & 23 \\
Records excluded & 0 & 5 \\
Records included & 1 & 18 \\
Level I clinical evidence & 0 & 0 \\
Level 2 clinical evidence & 2 & 18 \\
Level $\geq 3$ clinical evidence & 11 & 25 \\
Trials other than RCT & 11 & 25 \\
Case reports & 0 & 0 \\
Economic evidence & 0 & 3 \\
\hline
\end{tabular}

Notes: For definition of levels of evidence, see Core Evidence website (http://www. dovepress.com/core-evidence-journal).

Abbreviation: RCT, randomized clinical controlled trial.
Novel treatment options, such as thalidomide, bortezomib, and lenalidomide, are increasingly recognized as important and potent new therapies in overcoming resistant disease and contributing to improved outcome. ${ }^{8,9}$

\section{Epidemiology}

In the US, MM is the second most common hematologic malignancy after non-Hodgkin's lymphoma, with an estimated 19,920 new cases in 2008. ${ }^{10}$ This figure represents about $1.4 \%$ of all new cancer cases, including $14 \%$ of new hematologic malignancies. ${ }^{10}$ However, with a median survival in the order of three to four years, ${ }^{6}$ the disease claims a higher proportion of cancer-related deaths, estimated at 10,690 or $2 \%$ of all cancer deaths, including $20 \%$ of deaths due to hematologic malignancies. ${ }^{10}$

MM is predominantly a disease of older patients, with a mean age at diagnosis of 70 years. ${ }^{11}$ During 2000-2005, approximately $64 \%$ of diagnoses of MM were in people aged 65 years and older, and around 96\% were in people aged 45 years and older. Although age is the most significant risk factor for $\mathrm{MM}$, disease incidence is also higher among men than women (7.0 vs 4.6 per 100,000, respectively) and among African-Americans than Caucasian Americans (men: 14.4 vs 6.6 per 100,000, respectively; females: 9.8 vs 4.1 per 100,000 , respectively). ${ }^{11}$ The economic burden of MM has yet to be well described, but its high mortality and considerable antecedent morbidity is likely to make this substantial.

\section{Clinical features}

MM can be classified on the basis of symptoms, with symptomatic disease requiring evidence of related organor tissue-impairment, which is typically manifested by increased calcium, renal insufficiency, anemia, and/or bone lesions secondary to the plasma cell proliferative process. ${ }^{12}$ Other symptoms include bone pain, fatigue, fractures, recurrent infections, and weakness. Although the detection of immunoglobulin M-protein is characteristic, this is patient-specific and is absent in the $1 \%-2 \%$ of patients with nonsecretory $\mathrm{MM} .{ }^{4}$ In asymptomatic or smoldering MM, M-protein and/or bone marrow clonal cells are present, but there is no related organ- or tissue-impairment. Up to $25 \%$ of patients may have a smoldering pattern of disease at presentation. ${ }^{13}$

\section{Diagnosis}

A diagnosis of $\mathrm{MM}$ is often made incidentally during investigations of other conditions or as part of routine 
screening, as overt features of the disease may be absent. Assessment of serum and urine samples for M-proteins helps to establish a diagnosis, with immunofixation considered the gold standard when looking to confirm the presence of M-proteins and to distinguish heavy versus light chain types. ${ }^{12}$ Monoclonal gammopathies need to be excluded from polyclonal gammopathies because only the former are associated with neoplasia or potential neoplastic events. Serum protein electrophoresis is a suitable screening assay for M-protein whenever MM or related disorders are suspected, or in the presence of unexplained weakness, fatigue, anemia, infection, back pain, osteopenia, osteolytic lesions, or spontaneous fractures. ${ }^{12}$ Elevation of erythrocyte sedimentation rate, increased serum viscosity, hypergammaglobulinemia, hypercalcemia, Bence Jones proteinuria, renal insufficiency, and immunoglobulin deficiency (especially in the context of recurrent infection) may also be indicative and warrant screening for M-protein. Studies should include complete blood count, serum chemistry, bone marrow aspirate, and trephine biopsy for cytogenetic analysis of immunoglobulin translocations, as well as fluorescence in situ hybridization (FISH) and assessment of $\beta_{2}$-microglobulin, C-reactive protein, and lactate dehydrogenase. ${ }^{12}$

A diagnosis of MM requires M-protein levels of $\geq 30 \mathrm{~g} / \mathrm{L}$ and/or $\geq 10 \%$ or more plasma cells in the bone marrow. ${ }^{12}$
When these features are present together with related organ- or tissue-impairment, a diagnosis of symptomatic (versus asymptomatic) MM may be applied. Any patient with a serum M-protein level of $<30 \mathrm{~g} / \mathrm{L}$ and/or $<10 \%$ clonal plasma cells in the bone marrow in the absence of myeloma-related organ- or tissue-impairment is considered to have monoclonal gammopathy of undetermined significance (MGUS).

\section{Disease staging}

Two main staging systems are currently in use in MM, the International Staging System (ISS) and the Durie-Salmon system. ${ }^{6,14}$ The staging system most widely used since 1975 is the Durie-Salmon system, which is based on four clinical parameters that predict tumor burden: hemoglobin level; serum calcium level; number of bone lesions; and M-protein levels ${ }^{14}$ (Table 2). Serum creatinine level is additionally used to sub-categorize patients in each of the three stages according to renal function.

Although the Durie-Salmon system remains in widespread use, it is limited by observer dependence on assessments of the number of lytic lesions, by the characterization of new prognostic factors, and some redundancy. With respect to the latter, patients with stage I disease are not separated from those with smoldering myeloma in that neither group requires immediate treatment. ${ }^{15}$ Similarly, patients with either stage II or III disease

Table 2 The Durie-Salmon and International Staging System multiple myeloma staging systems ${ }^{6,14}$

\begin{tabular}{|c|c|c|}
\hline Stage & Durie-Salmon criteria & ISS criteria \\
\hline I & $\begin{array}{l}\text { All of the following: } \\
\text { - Hemoglobin }>10 \mathrm{~g} / \mathrm{dL} \\
\text { - Serum calcium normal or } \leq 12 \mathrm{mg} / \mathrm{dL} \\
\text { - Bone } x \text {-ray: normal bone structure (scale } 0 \text { ) or } \\
\text { solitary bone plasmacytoma only } \\
\text { - Low M-component production rate: } \operatorname{lgG}<5 \mathrm{~g} / \mathrm{dL} \text {; } \\
\operatorname{lgA}<3 \mathrm{~g} / \mathrm{dL} \text {; and Bence Jones protein }<4 \mathrm{~g} / 24 \text { hours }\end{array}$ & Serum $\beta_{2}-M<3.5 \mathrm{mg} / \mathrm{L}$ and serum albumin $\geq 3.5 \mathrm{~g} / \mathrm{dL}$ \\
\hline II & Neither stage I nor stage III & $\begin{array}{l}\text { Neither stage I nor stage III according to the following } \\
\text { subcategories: } \\
\text { - Serum } \beta_{2}-\mathrm{M}<3.5 \mathrm{mg} / \mathrm{L} \text { and serum albumin }<3.5 \mathrm{~g} / \mathrm{dL} \text {; } \\
\text { or } \\
\text { - Serum } \beta_{2}-\mathrm{M} 3.5-5.5 \mathrm{mg} / \mathrm{L} \text { irrespective of serum } \\
\text { albumin level }\end{array}$ \\
\hline III & $\begin{array}{l}\text { One or more of the following: } \\
\text { - Hemoglobin }<8.5 \mathrm{~g} / \mathrm{dL} \\
\text { - Serum calcium }>12 \mathrm{mg} / \mathrm{dL} \\
\text { - Advanced lytic bone lesions (scale } 3 \text { ) } \\
\text { - High M-component production rate: } \operatorname{lgG}>7 \mathrm{~g} / \mathrm{dL} \text {; } \\
\operatorname{lgA}>5 \mathrm{~g} / \mathrm{dL} \text {; Bence jones protein }>12 \mathrm{~g} / 24 \text { hours }\end{array}$ & Serum $\beta_{2}-M \geq 5.5 \mathrm{mg} / \mathrm{L}$ \\
\hline
\end{tabular}

Notes: *Durie-Salmon sub-classifications (either A or B):A: serum creatinine $<2.0 \mathrm{mg} / \mathrm{dL}$; and B: serum creatinine $\geq 2.0 \mathrm{mg} / \mathrm{dL}$. Abbreviations: $\beta_{2}-M, \beta_{2}$-microglobulin; Ig, immunoglobulin; ISS, International Staging System. 
typically have active, symptomatic myeloma. Moreover, with the recognition of the prognostic value of serum $\beta_{2}$-microglobulin and serum albumin, clinicians are increasingly complementing the Durie-Salmon system with the ISS. ${ }^{6}$

The ISS has been proposed as a simple, reliable, and more cost-effective predictor of survival in MM. ${ }^{6,15}$ Based on a collaboration involving investigators from 17 institutions worldwide and data on 11,171 previously untreated symptomatic myeloma patients, the ISS separates patients into three prognostic groups based on serum $\beta_{2}$-microglobulin and albumin levels at the time of starting initial systemic therapy (Table 2). The ISS has been validated by geographic region, by age ( $<65$ years versus $\geq 65$ years), by standard therapy versus autologous SCT, and in comparison with the Durie-Salmon and other staging systems. ${ }^{6,16}$

\section{Prognosis}

There is significant variation in the survival of patients with MM. Based on the ISS, the median survival of patients with stage I, II, or III disease is estimated at 62, 44, and 29 months, respectively. ${ }^{6}$ Although serum $\beta_{2}$-microglobulin and albumin levels combine in the ISS to provide a powerful prognostic tool, a number of independent prognostic markers have been described that may further assist in predicting outcome. ${ }^{17}$ Many established prognostic markers allowing identification of high-risk patients early in the disease course have been derived from studies of conventional chemotherapy and include age, $\beta_{2}$-microglobulin level, World Health Organization (WHO) performance status, serum calcium, interleukin-6 (IL-6) level, bone marrow plasma cell labeling index, and morphological features. ${ }^{18,19}$ However, in the current era of high-dose chemotherapy, novel immunomodulatory agents, and new small molecule inhibitors, a number of other prognostic markers relating to mechanisms of disease progression are now considered to be important. ${ }^{17}$

Abnormal cytogenetics play a dominant role in predicting the outcome of patients with acute leukemia, and the evidence now suggests that cytogenetics have a similar role in MM. Tricot and colleagues ${ }^{20,21}$ observed, using standard cytogenetic techniques, that in patients with newly diagnosed or previously treated disease, the presence of partial or complete deletions of chromosome 13 (del13q) and 11q abnormalities were associated with inferior event-free survival (EFS) and OS. In addition, they noted a significant association between the unfavorable karyotypes and immunoglobulin A ( $\operatorname{IgA}$ ) isotype, elevated levels of $\beta_{2}$-microglobulin, and age $>60$ years. $^{20}$

Conventional cytogenetic analysis is hampered by low mitotic activity of myeloma cells and may miss up to half of chromosome 13 abnormalities. Using FISH, Facon and colleagues ${ }^{22}$ demonstrated that in MM patients receiving first-line high-dose chemotherapy, the presence of chromosome 13 abnormalities was strongly predictive of poor survival, especially when associated with a $\beta_{2}$-microglobulin level of $\geq 2.5 \mathrm{mg} / \mathrm{L}$. FISH has since been used to identify patients with poor, intermediate, and better prognosis according to immunoglobulin heavy chain translocations and chromosome 13 abnormalities with other abnormalities such as $\mathrm{t}(4,14), \mathrm{t}(14,16)$, and del17q, emerging as prognostically unfavorable. ${ }^{23}$ However, as combinations of independent prognostic factors provide greater power than any one prognostic factor alone, the technique with potentially the highest utility in the future is gene expression profiling, which allows the simultaneous characterization of many different cytogenetic markers. ${ }^{24}$

\section{Evaluation of response}

Evaluation of tumor response to treatment is based on the assessment of changes in serum and/or urinary M-protein level. The most commonly used criteria for evaluating response are those introduced in 1998 by the European Group for Blood and Marrow Transplant (EBMT). ${ }^{4}$ The criteria for a complete response $(\mathrm{CR})$ require $<5 \%$ plasma cells in the bone marrow and the complete absence of M-protein by immunofixation and electrophoresis, with the response maintained for a minimum of six weeks. A partial response $(\mathrm{PR})$ is defined as a reduction in serum M-protein levels of $\geq 50 \%$ and a reduction in 24-hour urinary light chain excretion either by $\geq 90 \%$ or to $<200 \mathrm{mg}$, maintained for a minimum of six weeks. Near CR (nCR), a subset of PR, is defined as a $\mathrm{CR}$ with a positive immunofixation test but otherwise satisfies the criteria for $\mathrm{CR} .{ }^{25} \mathrm{~A}$ minimal response (MR) is defined as a reduction in serum M-protein levels of $25 \%-49 \%$ and a $50 \%-89 \%$ reduction in 24 -hour urinary light chain excretion that still exceeds $200 \mathrm{mg}$, maintained for a minimum of six weeks.

The International Myeloma Working Group (IMWG) has recently proposed changes to the original EBMT criteria in order to facilitate precise comparisons between new treatment strategies and to provide clarification of response in the clinical setting. ${ }^{26,27}$ For patients with measurable levels of serum and urine M-protein, the criteria for CR and PR remain unchanged. The most important changes are the inclusion of a new category of stringent $\mathrm{CR}$ ( $\mathrm{sCR}$ ) to reflect recent advances in therapy, and the inclusion of the serum-free light chain (FLC) assay to allow evaluation of patients with oligosecretory disease. The subcategories of 
nCR and very good PR (VGPR) have been integrated into a single category, VGPR, with sCR defined as CR based on EBMT criteria with the additional requirement for a normal FLC ratio and the absence of clonal cells in bone marrow by immunohistochemistry or immunofluorescence. VGPR is defined as serum and urine M-protein levels detectable by immunofixation, but not on electrophoresis, or a $\geq 90 \%$ reduction in serum $\mathrm{M}$-protein plus urinary $\mathrm{M}$-protein level $<100 \mathrm{mg}$ per 24 hours. The IMWG criteria eliminate the mandatory six-week period to confirm response and instead have a non-time-dependent confirmation for relapse and/or disease progression. ${ }^{26}$ Further modifications to this (including the restoration of MR as a response parameter associated with clinical benefit) as well as validation of key aspects, such as the assessment of serum FLC are anticipated. ${ }^{28}$

\section{Goals of therapy}

Treatment prolongs survival in MM, although remissions are inevitably followed by relapse. ${ }^{4}$ Therefore, the aim of treatment includes controlling disease by safely achieving a sequence of durable responses, without compromising quality of life. ${ }^{29}$ Given that current assessment techniques may not reflect true molecular remission, even using sCR or molecular CR criteria, and effective suppression of abnormal karyotype has been linked with long-term survival, suppression of abnormal karyotype may represent a part of the treatment goal to eradicate the myeloma clone. ${ }^{30}$ As the choice of therapy is influenced by patient factors, such as age and comorbidities, the goals of therapy are individual to the patient. Thus, CR may be the primary goal in a younger patient whereas control of disease activity to prevent progressive organ damage and to preserve performance status may be the goal in an older, more frail patient. The advent of novel therapies has dramatically expanded the options available for both younger and older patients in this context, especially given the favorable tolerability profiles seen with newer combinations, including bortezomib-based therapy as well as immunomodulatory approaches.

\section{Current therapy options}

Treatment recommendations for MM are dynamic and there is currently no single standard therapy for active myeloma. For patients with asymptomatic disease, a watch-and-wait approach is adopted because at present there is no evidence of benefit for early treatment in this population. ${ }^{31,32}$ Patients with symptomatic disease involving at least one of the following: hypercalcemia, renal insufficiency, anemia, or bone lesions require active treatment for which there are multiple options. ${ }^{12}$
These include proteasome inhibition (such as bortezomib), immunomodulating agents (such as thalidomide and lenalidomide), corticosteroids, bisphosphonates, conventional chemotherapy, radiotherapy, and autologous SCT.

\section{Newly diagnosed disease}

In patients with newly diagnosed disease who are eligible for autologous SCT, the initial goal of treatment is to reduce tumor burden with induction therapy. Induction regimens that are sufficiently nontoxic to hematopoietic stem cells include single-agent dexamethasone, combination vincristine + doxorubicin + dexamethasone (VAD), and novel regimens such as bortezomib-based treatments, thalidomide + dexamethasone, and lenalidomide + dexamethasone. ${ }^{7,27}$ More recent data suggest VAD has little or no role in induction given its inferiority to novel regimens demonstrated in numerous randomized trials. ${ }^{27}$ Following stem cell harvest, high-dose therapy is the standard of care for those undergoing autologous SCT given its survival advantage over conventional chemotherapy, ${ }^{33}$ which may involve a single autologous SCT, tandem autologous SCT, allogeneic SCT or syngeneic SCT. Interim data suggest there is no survival advantage of tandem over single autologous SCT, with the latter also being preferred over allogeneic SCT due to its superior efficacy in the absence of a syngeneic donor, its safety, and the absence of biological age-related disease differences. ${ }^{34}$ However, preliminary results for nonmyeloablative allogeneic transplantation are encouraging and support the feasibility of this approach. ${ }^{34}$ As almost all patients relapse, maintenance treatments that help prolong the duration of remission and survival are used, including thalidomide. ${ }^{35-37}$

Patients ineligible for SCT due to their age, performance status, comorbidities, or other factors have in the past received melphalan plus prednisone as the standard of care for induction therapy. ${ }^{38}$ However, other combinations have emerged, with the evidence base, in particular, supporting the combination of melphalan, prednisone, and thalidomide ${ }^{27,39}$ and most recently melphalan, prednisone, and bortezomib. ${ }^{40}$ Indeed, combination approaches with bortezomib as the first in class proteosome inhibitor, have shown particular promise both in autologous SCT eligible and nontransplantation populations, with high-quality responses seen. ${ }^{27}$ Other first-line options include melphalan, prednisone, and lenalidomide, ${ }^{41}$ lenalidomide plus dexamethasone, ${ }^{42,43}$ or dexamethasone plus thalidomide or bortezomib. ${ }^{39,44}$ The combination of lenalidomide and dexamethasone is now recognized by the National Comprehensive Cancer Network (NCCN) practice guidelines as an option for primary induction therapy in 
transplantation candidates based on category of evidence 2B (lower-level evidence including clinical experience and nonuniform consensus), ${ }^{27}$ together with bortezomib-based therapies. $^{27}$

\section{Relapsed or refractory disease}

An ongoing effort toward understanding the molecular pathogenesis of MM has led to the rational development of novel therapeutic agents, such as the immunomodulatory agents thalidomide and lenalidomide, and the proteasome inhibitor bortezomib, in this setting. The combination of these agents with dexamethasone in particular has shown impressive activity in relapsed or refractory $\mathrm{MM}$ and adds to the wide range of therapeutic options available..$^{45}$ Other options include conventional chemotherapy, melphalan plus prednisone, dexamethasone alone in good-risk patients and, in patients with early stem cell harvest, autologous SCT may be considered as salvage therapy. ${ }^{27}$

According to NCCN guidelines, patients who relapse after more than six months may benefit from reduction with the primary induction therapy. ${ }^{27}$ Conventional dose salvage therapy in combination with novel agents can be considered in patients with progressive disease following allogeneic or autologous SCT, in patients with primary progressive disease following initial allogeneic or autologous SCT, and in patients who are not candidates for transplantation with progressive or relapsing disease. Possible salvage therapies with category 1 evidence (uniform NCCN consensus based on high-level evidence) or 2A (uniform NCCN consensus based on lower-level evidence including clinical experience) are summarized in Figure 1, together with recommended options for induction and maintenance therapies. ${ }^{27}$ As an example, lenalidomide combined with dexamethasone has received US Food and Drug Administration (FDA) approval, based on two studies of 692 patients, for use in MM patients with at least one prior treatment and so is assigned a category 1 recommendation. ${ }^{46}$ The NCCN recommends anticoagulation therapy in patients treated with lenalidomide plus dexamethasone with lenalidomide monotherapy as a category $2 \mathrm{~A}$ recommendation. ${ }^{27}$

\section{Thalidomide}

As a salvage therapy for patients with relapsed or refractory MM, thalidomide has been investigated as monotherapy, in combination with dexamethasone (Thal + Dex), with bortezomib and dexamethasone, and in combination with dexamethasone, cisplatin, doxorubicin, cyclophosphamide, and etoposide. ${ }^{47-49}$ As a single-agent therapy, thalidomide has demonstrated an overall response rate (ORR) approaching

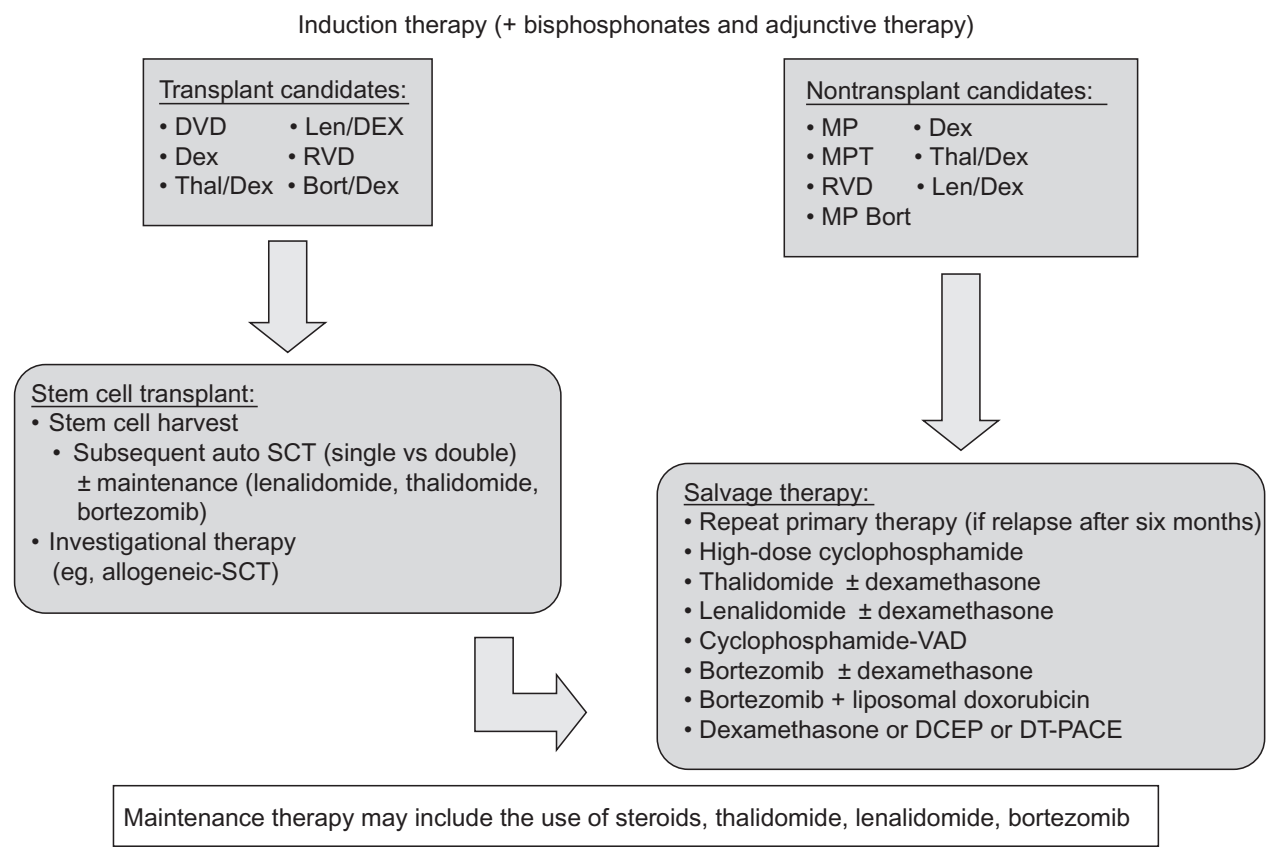

Figure I Treatment options in multiple myeloma. ${ }^{27}$

Abbreviations: Bort, bortezomib; Bort/Dex, bortezomib and dexamethasone; DCEP, dexamethasone, cyclophosphamide, etoposide, and cisplatin; Dex, dexamethasone; DT-PACE, dexamethasone, thalidomide, cisplatin, doxorubicin, cyclophosphamide, and etoposide; DVD, liposomal doxorubicin, vincristine, and dexamethasone; Len/Dex, lenalidomide plus dexamethasone; MP, melphalan and prednisone; MPT, melphalan, prednisone, and thalidomide; SCT, stem cell transplantation; RVD, lenalidomide, bortezomib and dexamethasone; VAD, vincristine, doxorubicin, and dexamethasone;Thal/Dex, thalidomide plus dexamethasone. 
$30 \%$, with a relatively low CR rate of $1.6 \%$, and an incidence of venous thromboembolism (VTE) of $3 \%$, and a rate of discontinuation due to intolerance of $15 \% .{ }^{50}$ The combination of thalidomide and dexamethasone offers significantly greater activity than respective single-agent therapies, with a rate of PR or better in the order of 55\%-59\% (CR 0\%-23\%), and a median survival of 13-26 months in relapsed or refractory disease. ${ }^{51-53}$ Low-dose thalidomide has been investigated in combination with cyclophosphamide and dexamethasone, yielding an ORR in one study of $79 \%$, including a CR rate of $17 \% .{ }^{54}$ Two-year OS and EFS were $73 \%$ and $34 \%$, respectively.

\section{Bortezomib}

Bortezomib was first studied in the setting of relapsed or refractory MM, and showed an overall response rate of $28 \%$ (PR or better) including $10 \% \mathrm{CR} / \mathrm{nCR}$ in heavily pretreated patients, ${ }^{25}$ leading to its accelerated approval by the FDA in 2003. In a recent systematic analysis, single-agent bortezomib was compared with single-agent thalidomide in patients with relapsed or refractory MM. ${ }^{55}$ The ORR was $41 \%$ for patients receiving bortezomib versus $22 \%$ for thalidomide. Similarly, bortezomib monotherapy yielded a higher ORR than single-agent dexamethasone in the relapse setting (38\% vs $18 \%$, respectively) and a higher $\mathrm{CR}$ rate $(6 \%$ vs $1 \%) .{ }^{56}$ Bortezomib was associated with improved TTP compared with single-agent dexamethasone (6.2 months vs 3.5 months, respectively) and one-year survival ( $80 \%$ vs $66 \%$ ). A recent update showed an ORR of $43 \%$ (PR or better) and a median OS of 29.8 months. ${ }^{57}$ There is also evidence showing increased response rates for bortezomib in combination with dexamethasone. ${ }^{25,58,59}$ In combination with low-dose melphalan and dexamethasone, bortezomib yielded an ORR of $69 \%$, including $29 \%$ with VGPR or better. ${ }^{60}$ The recent FDA approval of a novel bortezomib combination with pegylated liposomal doxorubicin was based on a priority review of interim data from a phase III clinical trial, which showed that this combination significantly extended TTP compared with bortezomib alone (9.3 months vs 6.5 months, respectively). OS was also significantly improved compared with bortezomib alone. ${ }^{61}$ Bortezomib is currently being investigated in the relapsed or refractory disease setting in combination with numerous novel agents, including tanespimycin (an inhibitor of heat-shock protein 90), perifosine (an AKT inhibitor), and oral vorinostat and related histone deacetylase inhibitors. ${ }^{57,62,64,65}$ Importantly, a four-drug combination has shown particular promise, with a phase I/II trial of bortezomib, melphalan, prednisone, and thalidomide yielding an ORR of $67 \%$ (all PR), including $43 \%$ with a VGPR. ${ }^{66}$

\section{Unmet needs}

Corticosteroids and alkylating agents have formed the mainstay of therapy for decades and continue to be used in combination regimens, where drugs with different mechanisms of action can offer important synergistic effects. However, more effective targeted therapies are beginning to emerge as a result of an improved understanding of the biology of MM. ${ }^{13}$ The rational development of these therapies, which include lenalidomide, thalidomide, and bortezomib, provides an opportunity to treat patients more effectively with fewer side-effects while aiming for durable responses. With mechanisms of action that are distinct from cytotoxic chemotherapies, these novel treatments (ORR) will continue to offer synergistic effects with conventional treatments and so offer potential survival benefit.

Thalidomide was the first immunomodulatory drug to demonstrate significant activity in newly diagnosed and relapsed disease, particularly in combination with dexamethasone. Its anti-MM effects are directed by multiple mechanisms that include antiangiogenesis, immunomodulation of the tumor microenvironment, and induction of apoptosis in tumor cells. ${ }^{49}$ However, in addition to having teratogenic potential, thalidomide is associated with many possible side effects, including sedation, fatigue, skin rash, and constipation; less common side effects include bradycardia, impotence, neutropenia, dysmenorrhea, and edema. Importantly, long term use can cause peripheral neuropathy. ${ }^{9}$ In addition to neuropathy, perhaps the most worrying side effect is VTE, including deep vein thrombosis (DVT), which is particularly problematic in combination with multiagent chemotherapy and dexamethasone. ${ }^{67,68}$

\section{Lenalidomide}

As a means of enhancing the immunomodulatory effects and overcoming the nonhematological adverse events of thalidomide, analogs such as lenalidomide have been developed. ${ }^{9,69}$ Like thalidomide, lenalidomide exerts pleiotropic effects, which include immunomodulatory, antiangiogenic, and antineoplastic activities. ${ }^{69}$ In preclinical studies, lenalidomide has demonstrated more potent anti-MM activity than its parent compound and its toxicity profile is more favorable. ${ }^{9,69}$ After comprehensive phase I and phase II trials in advanced MM, followed by two pivotal phase III trials, lenalidomide was approved by the US FDA and by the European Medicines Agency (EMEA) in June $2007^{70}$ for use in combination with dexamethasone in the treatment of MM in patients who have received at least one prior therapy.

\section{Mechanism of action in MM}

The molecular mechanisms associated with disease progression in $\mathrm{MM}$ are dependent on the interaction between MM 
cells and the bone marrow microenvironment. ${ }^{71}$ Briefly, the adhesion of MM cells to bone marrow stromal cells triggers the release of cytokines that mediate separate pathways of MM cell growth and survival, including proliferation, antiapoptosis, cell cycle progression, and migration. Stromal cell-derived IL-6, tumor necrosis factor-alpha (TNF- $\alpha$ ) and vascular endothelial growth factor (VEGF), for example, are involved in the activation of several MM cell signaling pathways, including phosphatidylinositol 3-kinase (PI3K)/Akt, Janus kinase (JAK)/signal transducer and activator of transcription 3 (STAT3), Raf/Mek/ mitogen-activated protein kinase (MAPK), and NF- $\mathrm{KB}$, together with their downstream targets (Figure 2). ${ }^{72}$

Lenalidomide has been shown to affect many of the interactions central to myeloma development by both direct and indirect mechanisms. ${ }^{73}$ The direct effects of lenalidomide include induction of apoptosis or cell cycle arrest of the tumor cell $1^{71,74,75}$ and indirect effects involving alteration of the tumor microenvironment and augmentation of the innate and acquired immune responses. Combined, these effects result in effective tumor cell reduction and suppression. This duality of action may be important in the treatment of MM. ${ }^{76}$
The rational development of lenalidomide as an anticancer agent followed the success of thalidomide, a potent inhibitor of TNF- $\alpha$ with antiangiogenesis activity and T-cell costimulatory activity. ${ }^{73}$ Compared with its parent compound, lenalidomide is a more powerful inhibitor of TNF- $\alpha$ secretion by activated monocytes. ${ }^{77,78}$ In addition to TNF- $\alpha$, lenalidomide also inhibits transforming growth factor-beta (TGF- $\beta$ ) and the proinflammatory cytokines, IL-1 $\beta$, IL-6, and IL-12, whereas secretion of the antiinflammatory cytokine IL-10 appears to be increased by lenalidomide. ${ }^{78,79}$ This differential regulation of cytokine activity, and particularly IL-6 activity, provides the basis for lenalidomide altering the bone marrow microenvironment in which the aberrant expression of proinflammatory cytokines is important for the growth and survival of MM cells. ${ }^{71}$ Moreover, inhibition of VEGF by lenalidomide may alter the bone marrow microvasculature, thereby making the tumor microenvironment less hospitable for MM cell growth, migration, and survival. ${ }^{71,78}$ VEGF inhibition likely occurs via the PI3K/Akt signaling pathway, which normally becomes phosphorylated in response to VEGF stimulation..$^{80,81}$

Lenalidomide is up to 2,000 times more potent than thalidomide in stimulating the proliferation of T-cells and

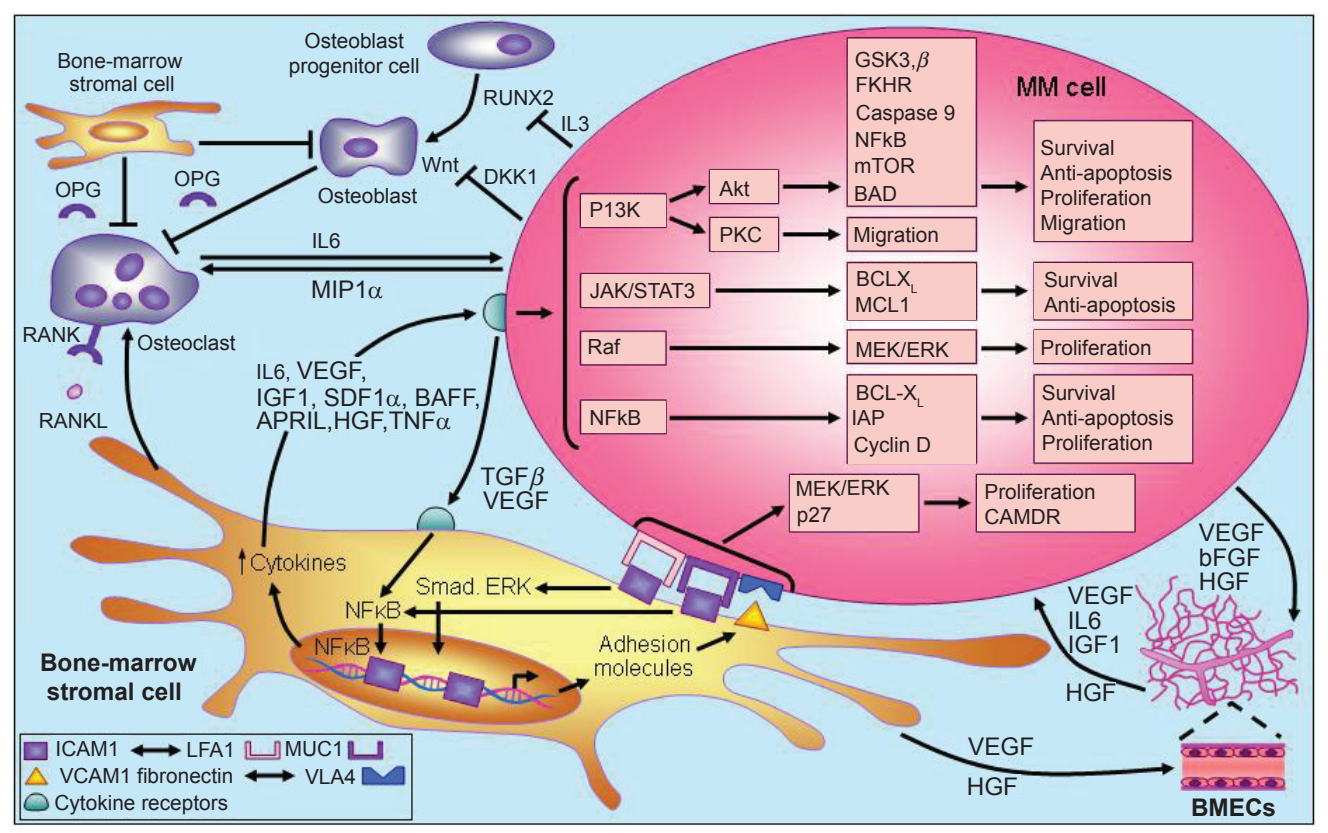

Figure 2 Pathogenesis of multiple myeloma. Copyright @ 2007. Adapted with permission from Macmillan Publishers Ltd. Hideshima T, Mitsiades C,Tonon G, et al Understanding multiple myeloma pathogenesis in the bone marrow to identify new therapeutic targets. Nat Rev Cancer. 2007;7:585-598.

Abbreviations: BAD, BCL-X associated death promoter; BCL- $X_{L}$, basal cell lymphoma-extra large; bFGF, basic fibroblast growth factor; BMECs, bone marrow endothelial cells; CAMDR, cell adhesion-mediated drug resistance; DKKI, Dickkopf-I; FKHR, forkhead transcription factor; GSK3 3 , glycogen synthase kinase 3-beta; HGF, hepatocyte growth factor; IAP, inhibitor of apoptosis proteins; ICAM, intercellular adhesion molecule; IGF-I, insulin-like growth factor; IL, interleukin;JAK/STAT, janus kinases/signal transducers, and activators of transcription; LFA, lymphocyte function-associated antigen; MCL, myeloid cell leukemia; MEK/ERK, mitogen-activated protein kinase/extracellular regulated kinase; MIPI $\alpha$, macrophage inflammatory protein Ialpha; mTOR, mammalian target of rapamycin; MUC, mucin; NFKB, nuclear factor kappa B; OPG, osteoprotegerin; PKC, protein kinase C; RANKL, receptor activated NFKB ligand; RUNX, runt-related transcription factor; SDFI $\alpha$, stromal cell derived factor I-alpha;TGF $\beta$, transforming growth factor beta; TNF- $\alpha$, tumor necrosis factor-alpha; VCAM, vascular cell adhesion molecule; VEGF, vascular endothelial growth factor; VLA4, very late antigen-4. 
up to 100 times more potent at increasing the release of IL-2 and interferon- $\gamma($ IFN- $\gamma){ }^{77}$ This T-cell costimulatory activity suggests that lenalidomide is able to act as an adjuvant to promote type 1 cell-mediated antitumor immune responses involving both $\mathrm{CD}^{+}{ }^{+} \mathrm{T}$-helper cells and $\mathrm{CD}^{+}$cytotoxic T-cells. ${ }^{73}$ The ability of lenalidomide to enhance activator protein-1 and NF- $\mathrm{KB}$ activity in antigen-primed T-cells has been proposed as a T-cell costimulatory mechanism, which may not only overcome T-cell anergy, but also potentiates any non-T-cell receptor-mediated signaling. ${ }^{78}$ In addition to bolstering the adaptive immune response, there is also evidence that lenalidomide can enhance innate immunity and natural killer cell-mediated lysis of MM cells in particular via its effects on IL-2 production by T-cells. ${ }^{71,73,82}$

Lenalidomide has been shown to directly potentiate apoptosis of MM cells via several pathways. These include inhibition of expression of the cellular inhibitor of apoptosis protein-2, potentiation of the activities of other apoptosis inducers such as TNF-related apoptosisinducing ligand (TRAIL), increased sensitivity to Fas induction, and enhanced caspase 8 activation. ${ }^{78}$ Caspase 8 , an integral component of Fas-mediated apoptosis, is sharply upregulated by lenalidomide (Figure 3). ${ }^{63}$ In addition, dexamethasone activates caspase 9 indicating that the two drugs in combination generate dual signals capable of enhanced cell death. ${ }^{71}$ Lenalidomide has been associated with direct antiproliferative activity against MM cells in the absence of immune cells or proapoptotic mechanisms by inducing $\mathrm{G}_{1}$ growth arrest. ${ }^{74,78}$ Importantly, lenalidomide inhibits the proliferation of malignant $\mathrm{B}$ cells while protecting normal CD34 ${ }^{+}$progenitor cells. ${ }^{75}$ The various mechanisms of action of lenalidomide are summarized in Figure 4.

\section{Clinical evidence for lenalidomide in MM}

\section{Newly diagnosed disease}

Lenalidomide is not yet approved for use in patients with previously untreated disease. However, several clinical studies have reported promising response and survival outcomes in this group of patients.

\section{Response rates and duration of response Lenalidomide plus dexamethasone}

In a phase III study, which had a planned enrollment of 500 patients with newly diagnosed MM but subsequently closed after 198 patients were enrolled due to external data affecting the acceptability of the control arm, patients were randomized to lenalidomide $25 \mathrm{mg}$ /day plus high-dose dexamethasone, or high-dose dexamethasone $40 \mathrm{mg}$ /day plus placebo. ${ }^{83}$ Lenalidomide was administered on 28 of 35 days for

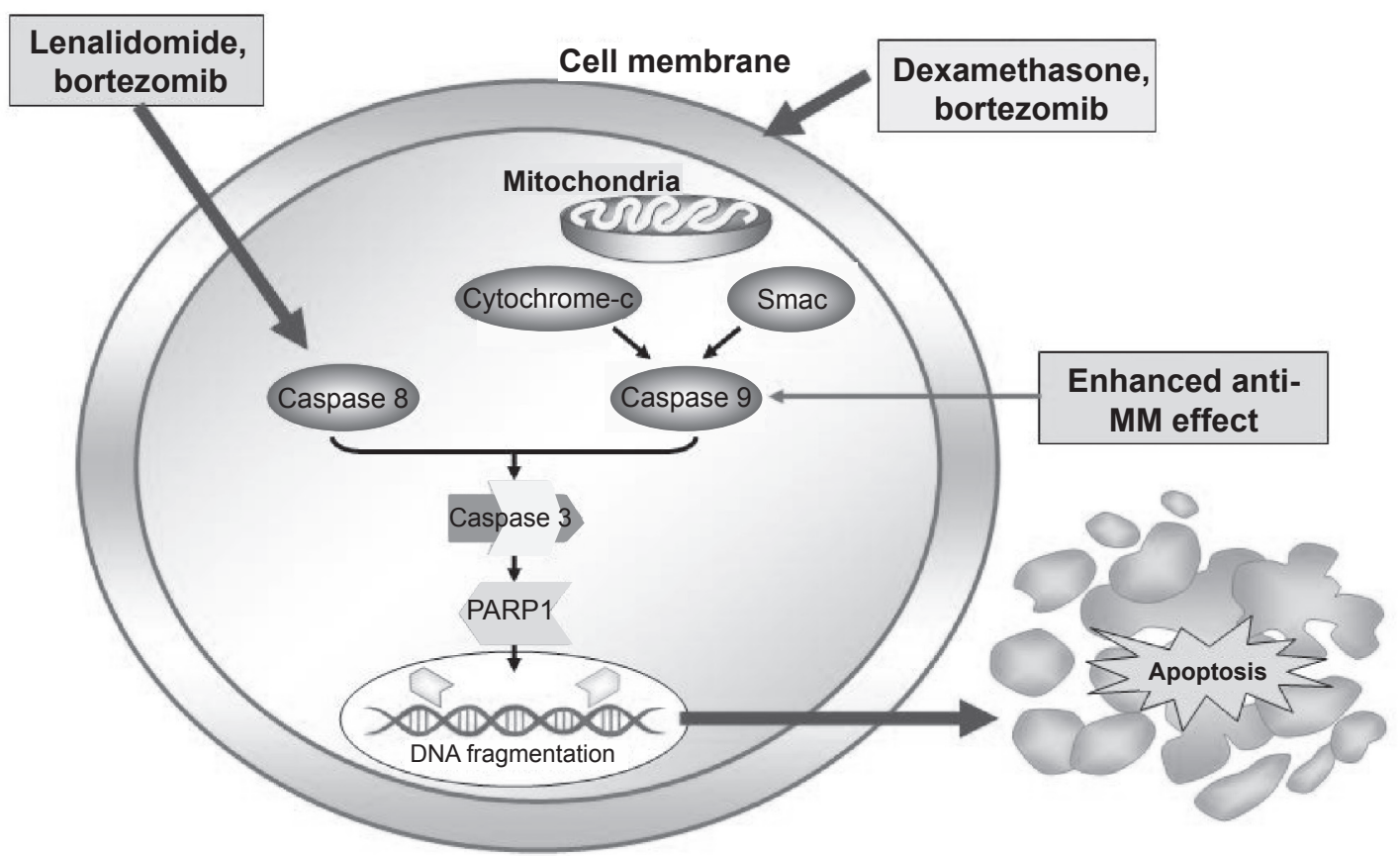

Figure 3 Caspase-mediated pathway. Copyright (C) 2007. Reproduced with permission by American Society of Hematology. Richardon P, Mitsiades C, Schlossman R, et al. The treatment of relapsed and refractory multiple myeloma. Hematology Am Soc Hematol Educ Program. 2007:317-323.

Abbreviations: MM, multiple myeloma; PARPI, Poly(adenine diphosphate-ribose) polymerase I; Smac, second mitochondria-derived activator of caspase; Caspase, cysteine-aspartic acid proteases. 


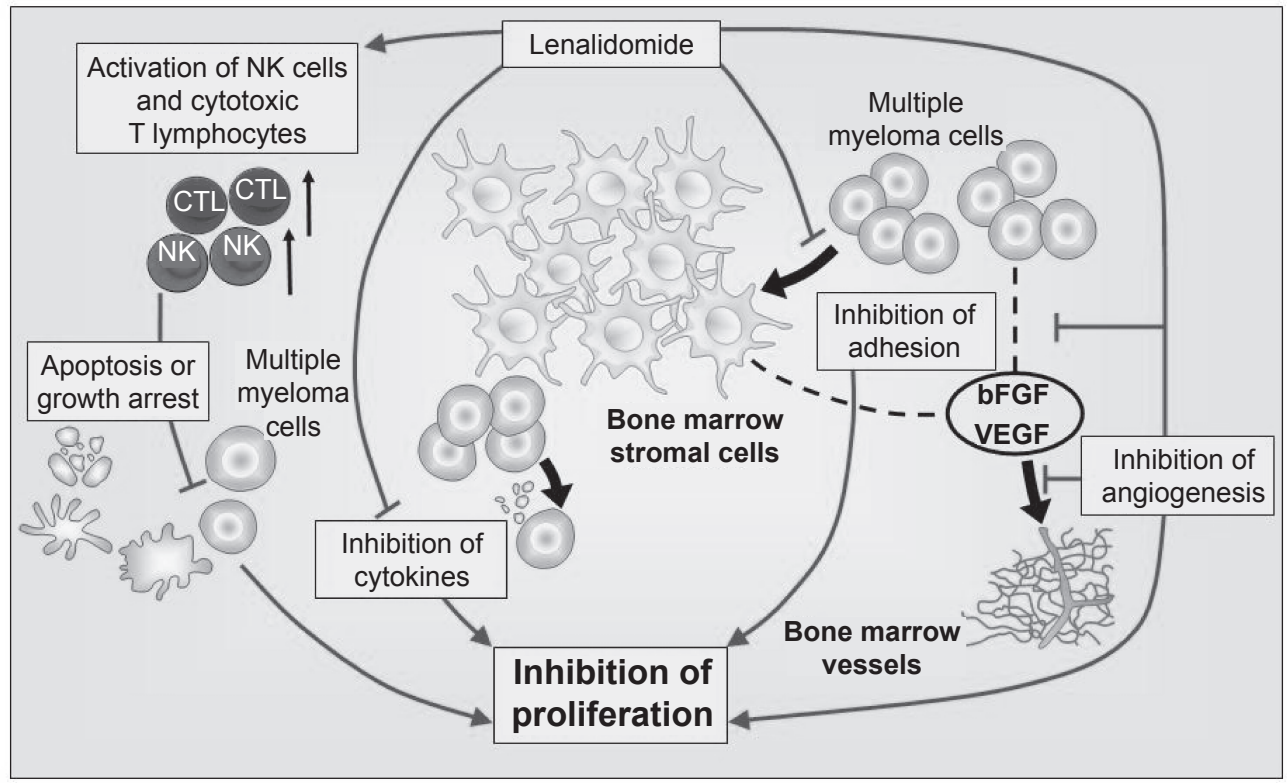

Figure 4 Mechanism of action of lenalidomide in multiple myeloma. Copyright (C) 2009. Adapted with permission from Richardson P, Jagannanth S, Hussein M, et al. Safety and efficacy of single-agent lenalidomide in patients with relapsed and refractory multiple myeloma. Blood. 2009; I 14:772-778.

Abbreviations: bFGF, basic fibroblast growth factor; CTL, cytotoxic T lymphocytes; NK, natural killer cells; VEGF, vascular endothelial growth factor.

three induction cycles, and then 21 of 28 days as maintenance thereafter. High-dose dexamethasone was administered on days 1-4, 9-12, and 17-20 during induction, and then days 1-4 and 15-18 during maintenance. Treatment with lenalidomide plus high-dose dexamethasone yielded an ORR of $85.3 \%$ and a CR rate of $22.1 \%$ versus treatment with high-dose dexamethasone alone $(51.3 \%$ and $3.8 \%$, respectively; $P=0.001)$.

A second phase III randomized study compared lenalidomide plus high-dose dexamethasone with lenalidomide plus low-dose dexamethasone in 445 patients with newly diagnosed MM. ${ }^{84}$ Lenalidomide was dosed at $25 \mathrm{mg} /$ day on days 1-21 every 28 days. Patients in the high-dose arm received dexamethasone $40 \mathrm{mg}$ /day on days $1-4,9-12$, and 17-20 every 28 days, whereas patients in the low-dose arm received dexamethasone $40 \mathrm{mg}$ /day on days $1,8,15$, and 22 every 28 days. Within the first four cycles of treatment, a response of $\mathrm{PR}$ or higher was seen in $82 \%$ of patients treated with lenalidomide plus high-dose dexamethasone versus $70 \%$ of patients in the lenalidomide plus low-dose dexamethasone $\operatorname{arm}(P=0.007)$. CR plus VGPR rates were $52 \%$ versus $42 \%$, respectively $(P=0.06)$.

In a phase II study, 34 previously untreated MM patients (mean age 64 years) were administered lenalidomide $25 \mathrm{mg} /$ day on days 1-21 of a 28-day cycle and dexamethasone $40 \mathrm{mg}$ /day on days $1-4,9-12$, and 17-20 of each cycle for at least four cycles. ${ }^{42}$ Treatment with lenalidomide plus dexamethasone yielded an TTP of $91 \%$, including six patients (18\%) with a $\mathrm{CR}$ and 13 (38\%) who met the criteria for VGPR and nCR. ${ }^{42,43}$
Among 21 patients who did not subsequently receive SCT and were eligible for treatment beyond four cycles at the discretion of the investigator, 14 (67\%) achieved either a CR or VGPR. ${ }^{43}$

\section{Lenalidomide/cyclophosphamide/dexamethasone}

A phase II study of 33 patients (median age 63 years) with newly diagnosed MM evaluated the combination of lenalidomide $25 \mathrm{mg}$ /day on days 1-28 of every 28 -day cycle, cyclophosphamide $300 \mathrm{mg} / \mathrm{m}^{2}$ on days 1,8 , and 15 of each cycle, and dexamethasone $40 \mathrm{mg} /$ day on days $1,8,15$, and 22 of each cycle. ${ }^{85}$ Among 19 of 33 evaluable patients, two achieved a VGPR (10.5\%) and 13 achieved a PR (68.4\%), giving an ORR of $78.9 \%$.

\section{Lenalidomide/bortezomib/dexamethasone (RVd)}

In a phase I/II study, the combination of lenalidomide $15-25 \mathrm{mg} /$ day on days $1-14$, bortezomib $1.0-1.3 \mathrm{mg} / \mathrm{m}^{2}$ on days $1,4,8$, and 11 , and dexamethasone $40 / 20 \mathrm{mg} /$ day (cycles 1-4/5-8) on day of and day after bortezomib administration for up to eight 21-day cycles produced an ORR of $98 \%$ in 42 evaluable patients with newly-diagnosed MM. ${ }^{86}$ Nine of 42 (21\%) patients had a CR, 3 (7\%) had nCR, $10(24 \%)$ had VGPR, and 19 (45\%) had PR, giving an ORR of $98 \%$ at the time of this analysis. All 11 patients who received treatment with lenalidomide/bortezomib/ dexamethasone RVd at the phase II dose level of lenalidomide $25 \mathrm{mg}$, bortezomib $1.3 \mathrm{mg}$, and dexamethasone $20 \mathrm{mg}$ achieved PR or better (100\% ORR). 
Clarithromycin/lenalidomide/dexamethasone (BiRD)

In a phase II study of 72 patients (median age 63 years) with newly diagnosed $\mathrm{MM}$, induction therapy with clarithromycin $500 \mathrm{mg}$ twice -daily, lenalidomide $25 \mathrm{mg}$ /day on days 1-21 of a 28-day cycle, and dexamethasone $40 \mathrm{mg}$ /day once weekly was associated with an objective response of PR or better in $65(90.3 \%)$ patients $(90.3 \%)$, including a CR rate of $38.9 \% .{ }^{87}$ Fifty-three patients (73.6\%) achieved at least a 90\% decrease in M-protein levels. The mean duration of response was 333 days and the mean time to response was 54 days, with a mean time to maximum response of 209 days. Patients with atypical serum immunofixation pattern (ASIP) development during induction therapy with BiRD had significantly better response than patients without ASIP, with a $\mathrm{CR}$ rate of $71 \%$ versus $23 \%$, respectively $(P=0.00002) .{ }^{88}$

\section{Lenalidomide/melphalan/prednisone (RMP)}

In a phase I/II study conducted by the Italian Multiple Myeloma Network, nine monthly cycles of lenalidomide $5-10 \mathrm{mg} /$ day administered on days $1-21$, melphalan $0.18-0.25 \mathrm{mg} / \mathrm{kg}$ given on days $1-4$, and prednisone $2 \mathrm{mg} / \mathrm{kg}$ given on days $1-4$ yielded an ORR of $81 \%$ in 53 elderly patients (median age 71 years) with newly diagnosed MM. ${ }^{41}$ Seven patients $(13 \%)$ in total had a CR, including 5 of $21(24 \%)$ patients assigned to lenalidomide $10 \mathrm{mg}$ plus melphalan $0.18 \mathrm{mg} / \mathrm{kg}$, and $2(10 \%)$ of $20(10 \%)$ patients assigned to lenalidomide $10 \mathrm{mg}$ plus melphalan $0.25 \mathrm{mg} / \mathrm{kg}$. Another 13 patients (25\%) in total had a VGPR. The median time to best response was four months and PR was achieved in $53 \%$ of patients after the first cycle of treatment.

\section{Time to progression}

\section{Lenalidomide plus dexamethasone}

In a phase II study, lenalidomide plus dexamethasone was associated with a median TTP of 32.4 months in patients who did not undergo SCT, whereas median TTP was not reached at the time of publication in patients who underwent SCT. ${ }^{43}$ The two-year TTP rates were $71 \%$ for the entire cohort, $66 \%$ in the nontransplantation group, and $83 \%$ in the transplantation group.

\section{RVd}

In a phase I/II study, median TTP was not reached after a median follow-up of four months in 42 patients who received lenalidomide in combination with bortezomib and dexamethasone. ${ }^{86}$

\section{RMP}

Among 21 elderly patients (median age 69 years) in a phase I/II study who received the maximum tolerated dose of lenalidomide $10 \mathrm{mg} /$ day for 21 days, melphalan $0.18 \mathrm{mg} / \mathrm{kg}$ for four days, and prednisone $2 \mathrm{mg} / \mathrm{kg}$ for four days of every 28 days for a maximum of nine cycles, followed by lenalidomide $10 \mathrm{mg}$ /day for 21 of every 28 days as maintenance after a median follow-up of 29.5 months. The median TTP was 28.5 months. ${ }^{89}$

\section{Overall survival}

Lenalidomide plus dexamethasone

In a phase III study comparing lenalidomide in combination with either high-dose or low-dose dexamethasone, OS was superior for the low-dose dexamethasone combination $(P=0.006){ }^{84}$

\section{RVd}

In a phase I/II study, median OS was not reached after a median follow-up of four months. ${ }^{86}$

\section{BiRD}

Among 72 evaluable patients treated with BiRD in a phase II study, actuarial EFS at two years was $97.2 \% .{ }^{87}$ The median EFS duration was not yet reached.

\section{One-, two-, and three-year survival}

\section{Lenalidomide plus dexamethasone}

Lenalidomide plus dexamethasone has recently been evaluated in a randomized controlled phase III study of 445 patients with previously untreated MM. ${ }^{84,90}$ Survival significantly favored lenalidomide plus low-dose dexamethasone, with a one-year survival rate of $96 \%$ compared with $88 \%$ for lenalidomide plus high-dose dexamethasone $(P=0.006) .{ }^{84}$ Among patients aged $<65$ years, one-year survival rates for low- versus high-dose dexamethasone were $97 \%$ versus $92 \%$, respectively $(P=0.022)$; the respective data for patients aged $\geq 65$ years were $94 \%$ versus $83 \%(P=0.002) .{ }^{90}$ Two-year OS rates were $87 \%$ versus $75 \%$, respectively. ${ }^{84}$ In a landmark analysis of the 210 patients who were alive and went off study after four months, the one- and two-year OS rates among the 102 patients who underwent SCT were 99\% and $94 \%$, respectively. In contrast, among the 108 patients who did not undergo SCT, one- and two-year OS rates were $85 \%$ and $70 \%$, respectively.

In a second randomized controlled phase III study, one-year OS rates were $93 \%$ and $91 \%$ in patients assigned to lenalidomide plus high-dose dexamethasone and highdose dexamethasone alone, respectively. ${ }^{83}$ In a subgroup analysis that considered patients with and without abnormal karyotypes at baseline, one-year OS rates among those with abnormal karyotypes were $82 \%$ and $77 \%$ in patients treated 
with lenalidomide plus high-dose dexamethasone and dexamethasone alone, respectively. ${ }^{91}$

Among a cohort of 34 patients treated with lenalidomide plus dexamethasone in a phase II study, two- and three-year OS was approximately $91 \%$ and $88 \%$, respectively. ${ }^{43}$

\section{RMP}

Among 53 elderly patients treated with RMP in a phase II study, the one-year OS rate was $100 \%{ }^{41}$ Among 21 patients treated with the maximum tolerated dose in this study followed by lenalidomide $10 \mathrm{mg}$ /day on 21 of every 28 days as maintenance therapy, the two-year OS rate was $90.5 \% .{ }^{92}$

\section{Adverse events}

Lenalidomide plus dexamethasone

A phase III study conducted by the Eastern Cooperative Oncology Group (ECOG) reported a lower rate of grade 3 or 4 adverse events among patients who were randomized to lenalidomide plus low-dose dexamethasone than in patients randomized to lenalidomide plus high-dose dexamethasone..$^{90}$ In patients assigned to high- versus low-dose dexamethasone, major grade 3 or 4 toxicities and their respective rates were: neutropenia (10\% vs $19 \%$, respectively; $P=0.01)$; VTE $(25 \%$ vs $9 \% ; P<0.001)$; and infection/pneumonia ( $16 \%$ vs $6 \% ; P<0.001)$. Grade 3 or 4 nonhematological toxicities occurred in $49 \%$ and $32 \%$ of patients assigned to high- versus low-dose dexamethasone, respectively in combination with lenalidomide $(P<0.001)$. Of verified deaths in the high-dose dexamethasone arm, 13 were due to disease progression, six cases were related to VTE, three were due to infection, and another five cases were due to cardiac ischemia, stroke, and respiratory failure. Of nine verified deaths in the low-dose dexamethasone arm, five were due to disease progression, two to infection, one to VTE, and one to cardiac arrest. In the first four months of therapy, the mortality rate was $5 \%$ in the high-dose dexamethasone group compared with $0.5 \%$ in the low-dose group.

In a second randomized, double-blind, phase III study, lenalidomide plus high-dose dexamethasone was associated with a higher rate of adverse events than treatment with high-dose dexamethasone alone. ${ }^{83}$ Grade 3 or 4 neutropenia was reported by $13.5 \%$ of patients treated with lenalidomide plus high-dose dexamethasone compared with $2.4 \%$ of patients treated with high-dose dexamethasone alone $(P=0.01)$. There were $20 \mathrm{VTE}$ events in the lenalidomide plus dexamethasone group including 14 events associated with aspirin prophylaxis; there were 12 thromboembolic events in the dexamethasone-only group all of which were associated with aspirin prophylaxis.

In phase II studies of lenalidomide plus dexamethasone, $47 \%-55 \%$ of patients experienced a grade 3 or 4 nonhematological toxicity during therapy, most commonly fatigue $(15 \%-21 \%)$, anxiety (6\%), pneumonitis (6\%), muscle weakness $(6 \%)$, and rash $(6 \%) .{ }^{42,43}$ Grade 3 or 4 hematological adverse events included neutropenia (12\%-21\%), leucopenia (9\%), lymphopenia (6\%), and anemia (6\%). All patients received aspirin once daily as thromboprophylaxis. However, although one patient developed a grade 4 pulmonary embolism they recovered with therapy. Two patients died from infection that was deemed to be possibly related to study therapy. ${ }^{42,43}$

\section{RVd}

In a phase I/II dose-finding study, among 53 evaluable patients who completed a median of six treatment cycles, 14 patients discontinued treatment. ${ }^{86}$ Two dose-limiting toxicities of grade 3 hyperglycemia due to high-dose dexamethasone were seen at dose level 4 (lenalidomide $25 \mathrm{mg} /$ day, bortezomib $1.3 \mathrm{mg} / \mathrm{m}^{2}$, and dexamethasone $40 \mathrm{mg} /$ day), with subsequent recruitment into phase II involving a reduction in dexamethasone dose to $20 \mathrm{mg} /$ day. Dose reductions in cycle 2 and beyond occurred for lenalidomide in 12 patients, bortezomib in 11 patients, and dexamethasone in 18 patients Adverse events were manageable with no unexpected events, no grade 4 peripheral neuropathy, two episodes of DVT, and no treatment-related mortality.

\section{BiRD}

In a phase II study, 17 of 72 patients treated with BiRD required at least one lenalidomide dose reduction for a grade 3 or 4 adverse event. ${ }^{87}$ Grade 3 or 4 hematological toxicities included neutropenia (19.4\%), anemia (13.8\%), and thrombocytopenia (22.2\%). Nonhematological grade 3 or 4 toxicities included myopathy $(11.1 \%)$, thrombosis $(9.7 \%)$, rash $(5.6 \%)$, and diverticular abscess $(5.6 \%)$. VTE occurred in nine patients $(12.5 \%)$, of which five events were associated with aspirin interruption or poor compliance. ${ }^{87}$

\section{RMP}

In a phase II study of RMP in 53 elderly patients, at the maximum tolerated dose, grade 3 or 4 hematological toxicities were neutropenia (52\%), thrombocytopenia (24\%), and anemia (5\%). ${ }^{41}$ Grade 3 febrile neutropenia, vasculitis, and VTE were reported in $10 \%, 10 \%$, and $5 \%$ of patients, respectively. In a subgroup of 21 patients who were followed for a median of 29.5 months, grade 3 and 4 neutropenia 
were reported in $38 \%$ and $14 \%$ of patients, respectively, during initial therapy. ${ }^{92}$ Grade 3 and 4 thrombocytopenia were reported in $14 \%$ and $10 \%$ of patients, respectively. Whereas the incidence and depth of neutropenia did not increase with the number of cycles, thrombocytopenia was more pronounced after nine cycles. One patient required a lenalidomide dose reduction for severe neutropenia and three patients discontinued due to severe thrombocytopenia and neutropenia.

\section{Stem cell transplantation \\ Stem cell collection \\ Lenalidomide plus dexamethasone}

In MM patients who received initial therapy with lenalidomide plus dexamethasone, a retrospective analysis of a five-year treatment period at a single institution indicated there was a trend towards decreased peripheral blood stem cell yield with increasing duration of lenalidomide therapy. ${ }^{93}$ A retrospective study by Paripati and colleagues comparing lenalidomide plus dexamethasone induction therapy versus other induction therapy showed that the first attempt at stem cell collection was unsuccessful significantly more frequently in lenalidomide plus dexamethasone recipients compared with those who had received other induction therapy ( $7 \%$ vs $45 \%$, respectively; $P=0.001) .{ }^{94}$ Lenalidomide plus dexamethasone recipients had lower mean peripheral blood CD34+ cell counts compared with those who received other induction therapies ( 14.0 cells $/ \mu \mathrm{L}$ vs 28.9 cells $/ \mu \mathrm{L} ; P<0.0002)$ and mean total stem cells collected $\left(5.1 \times 10^{6}\right.$ cells $/ \mathrm{kg}$ vs $7.4 \times 10^{6}$ cells $/ \mathrm{kg}$; $P=0.0025)$ compared with those who received other induction therapies. However, compared with single-agent dexamethasone, thalidomide plus dexamethasone or vincristine/adriamycin/dexamethasone, there was no effect on quality of yield in patients receiving lenalidomide based on similar engraftment. ${ }^{93}$

\section{Lenalidomide-based induction therapy}

In a recent study where 21 patients with MM received lenalidomide-based induction therapy prior to stem cell mobilization, lenalidomide did not prevent the harvest of adequate numbers of $\mathrm{CD}^{+} 4^{+}$cells for autologous SCT (median 6.3 cells $\times 10^{6} / \mathrm{kg}$; range $2.4-19.7$ cells $\times 10^{6} / \mathrm{kg}$ ). ${ }^{95}$ Patients were mobilized with cyclophosphamide plus granulocyte colonystimulating factor $(\mathrm{G}-\mathrm{CSF})(\mathrm{n}=17), \mathrm{G}-\mathrm{CSF}$ and AMD3100 $(n=2)$, or G-CSF alone $(n=2)$. Repeat mobilization was required in patients who received G-CSF alone and was successful on the second attempt with the addition of AMD3100. The median number of collections was 3 (range 1-8) in patients mobilized with cyclophosphamide plus G-CSF and 4.5 (range 2-6) in those mobilized with G-CSF plus AMD3100. The respective median CD34 ${ }^{+}$ cell counts were $6.3 \times 10^{6} / \mathrm{kg}$ (range $3.0-19.7 \times 10^{6} / \mathrm{kg}$ ) and $8.4 \times 10^{6} / \mathrm{kg}$ (range 5.6-12.3 $\times 10^{6} / \mathrm{kg}$ ). No correlation between the number of lenalidomide cycles (median 4 , range 1-16) and the number of stem cell collections or total CD $34^{+}$cell counts was reported.

\section{BiRD plus G-CSF or G-CSF plus cyclophosphamide} for stem cell mobilization

In a subset of 28 treatment-naïve MM patients who were treated with the BiRD regimen in a phase II trial, the effect of cyclophosphamide plus G-CSF as a stem cell mobilization regimen compared with G-CSF alone was investigated. ${ }^{96}$ Successful stem cell harvest sufficient for two autologous SCTs was achieved in all patients who received mobilization with cyclophosphamide plus G-CSF, compared with only $33 \%$ of patients who were mobilized with G-CSF alone $(P<0.0001)$. No correlation between duration of lenalidomide and stem cell collection was observed.

\section{Response}

\section{Bortezomib/doxorubicin/dexamethasone followed}

by lenalidomide and prednisone

In a phase II study, 94 patients aged 65-75 years with newly diagnosed MM were treated with bortezomib and doxorubicin plus dexamethasone (PAD) induction (bortezomib $1.3 \mathrm{mg} / \mathrm{m}^{2}$ on days $1,4,8$, and 11 , pegylated liposomal doxrorubicin $20 \mathrm{mg} / \mathrm{m}^{2}$ on day 4 , and dexamethasone 40 $\mathrm{mg}$ on days $1-4,8-11$, and $15-18$ for cycle 1 and days $1-4$ for cycles 2-4) prior to reduced intensity autologous SCT. ${ }^{89}$ Cyclophosphamide $3 \mathrm{mg} / \mathrm{m}^{2}$ plus G-CSF was used to harvest stem cells, with patients then conditioned with tandem melphalan $100 \mathrm{mg} / \mathrm{m}^{2}$ and stem cell support (MEL100). Following autologous SCT, patients received consolidation therapy with lenalidomide $25 \mathrm{mg}$ /day on days $1-21$ plus prednisone $50 \mathrm{mg} /$ day every other day, and then maintenance therapy with lenalidomide alone $(10 \mathrm{mg} /$ day on days 1-21 every 28 days). After four cycles of PAD therapy, 96\% of patients had at least PR (60\% had at least VGPR, 23\% had at least nCR, and 13\% had CR), after tandem MEL100, $95 \%$ had at least PR (80\% at least VGPR, 60\% at least nCR, and $33 \%$ had CR), and after lenalidomide plus prednisone consolidation all patients had at least PR (89\% had at least VGPR, 78\% had at least $\mathrm{nCR}$, and 56\% had CR). 


\section{Adverse events}

Lenalidomide plus prednisone consolidation therapy

In a study of 94 elderly patients with newly diagnosed MM who received lenalidomide plus prednisone as consolidation therapy following PAD induction therapy and autologous SCT, one case of DVT and one discontinuation because of prolonged thrombocytopenia and anemia were reported during consolidation therapy. ${ }^{89}$

\section{Relapsed or refractory disease}

Two multicenter, double-blind, randomized, placebocontrolled phase III studies (MM-009/ and MM-010) investigated the efficacy and safety of lenalidomide plus dexamethasone versus dexamethasone alone in the treatment of patients with relapsed or refractory MM; they provided the basis for the approval of lenalidomide in this indication. ${ }^{46}$ Patients with relapsed or refractory MM and $\leq 3$ previous regimens were eligible. Based on the findings of phase I and II studies, lenalidomide was administered at $25 \mathrm{mg}$ /day on days 1-21 of each 28-day cycle. Patients were randomized to either four 28-day cycles of lenalidomide plus high-dose dexamethasone $40 \mathrm{mg}$ /day on days 1-4, 9-12, and 17-20 of each cycle, or matched placebo plus dexamethasone as for the group assigned to active treatment. After four cycles of therapy, treatment was continued until disease progression, but with dexamethasone $40 \mathrm{mg}$ administered only on days $1-4$ of every 28 -day cycle. The primary end point of TTP was evaluated according to EBMT criteria. ${ }^{4} \mathrm{~A}$ total of 353 patients in MM-009 and 351 patients in MM-010 were randomized and received study medication.

\section{Response rates and duration of response Lenalidomide plus dexamethasone}

In the MM-009 and MM-010 studies, lenalidomide in combination with high-dose dexamethasone led to a significantly better ORR compared with dexamethasone alone., ${ }^{2,3}$
In these studies, 108 patients $(61.0 \%)$ in MM-009 and 106 patients (60.2\%) in MM-010 assigned to lenalidomide plus dexamethasone achieved a response of PR or better. In comparison, 35 patients (19.9\%) in MM-009 and 42 patients $(24.0 \%)$ in MM-010 assigned to dexamethasone alone had a response to therapy $(P<0.001$ versus lenalidomide plus dexamethasone). In both studies, the $\mathrm{CR}$ rate in response to lenalidomide plus dexamethasone was approximately 15\% (MM-009: 14.1\%; MM-010: 15.9\%) and the nCR rate was approximately 9\% (MM-009: 10.2\%; MM-010: 8.5\%) (Table 3). ${ }^{2,3}$ In a pooled analysis that included data from all 704 patients enrolled in both trials, the ORR in the lenalidomide plus dexamethasone group and the dexamethasone-only group was $60.6 \%$ and $21.9 \%$, respectively $(P<0.001) .{ }^{97}$ The respective data for CR rate were $15.0 \%$ and $2.0 \%$ $(P<0.001)$. Among patients who received lenalidomide plus dexamethasone, the median duration of response was significantly higher for those who achieved a CR or nCR compared with those who achieved a PR (not yet reached vs 8.8 months; $P<0.001) .{ }^{98}$

Patients in the MM-009 and MM-010 studies were stratified according to $\beta_{2}$-microglobulin $(\leq 2.5 \mathrm{mg} / \mathrm{L}$ vs $>2.5 \mathrm{mg} / \mathrm{L}$ ), prior SCT (none vs $\geq 1$ ), and number of prior regimens ( $1 \mathrm{vs}>1)^{2,3}$ In both studies lenalidomide plus dexamethasone was associated with significantly higher response rates than dexamethasone alone, irrespective of $\beta_{2}$-microglobulin level, prior SCT, or number of prior therapies (Table 4). In addition, lenalidomide plus dexamethasone yielded higher response rates than dexamethasone alone irrespective of prior bortezomib or thalidomide therapy., 2,3

In a prospective, pooled subgroup analysis of 704 patients enrolled in the MM-009/ and MM-010 studies, the ORR was significantly higher with lenalidomide plus dexamethasone treatment compared with dexamethasone alone in patients who had received prior thalidomide $(60 \%$ vs $18 \%$, respectively; $P<0.01$ ) or in patients who had not had prior

Table 3 Treatment response, time to progression and overall survival in MM-009 and MM-0I02,3

\begin{tabular}{|c|c|c|c|c|c|c|}
\hline & \multicolumn{3}{|c|}{ MM-009 $(N=353)$} & \multicolumn{3}{|c|}{ MM-0IO $(\mathbf{N}=35 I)$} \\
\hline & Leni + Dex & Dex & $P$ value & Leni + Dex & Dex & $P$ value \\
\hline Randomized, n & 177 & 176 & & 176 & 175 & \\
\hline ORR (CR, nCR, PR), \% & 61.0 & 19.9 & $<0.00 \mathrm{I}$ & 60.2 & 24.0 & $<0.001$ \\
\hline $\mathrm{CR}, \%$ & 14.1 & 0.6 & $<0.001$ & 15.9 & 3.4 & $<0.001$ \\
\hline Median TTP, months & 11.1 & 4.7 & $<0.001$ & 11.3 & 4.7 & $<0.001$ \\
\hline Median OS, months & 29.6 & 20.2 & $<0.001$ & NR & 20.6 & 0.03 \\
\hline
\end{tabular}

Abbreviations: CR, complete response; Dex, placebo plus dexamethasone; Leni + Dex, lenalidomide plus dexamethasone; nCR, near complete response; NR, not reached; ORR, overall response rate; OS, overall survival; PR, partial response; TTP, time-to-progression. 
Table 4 Treatment outcomes in MM-009 and MM-0I0: subgroup analyses ${ }^{2,3}$

\begin{tabular}{|c|c|c|c|c|c|c|}
\hline \multirow[t]{2}{*}{ Subgroup analysis } & \multicolumn{3}{|c|}{ MM-009 (N = 353) } & \multicolumn{3}{|c|}{$M M-010(N=351)$} \\
\hline & Leni + Dex & Dex & $P$ value & Leni + Dex & Dex & $P$ value \\
\hline \multicolumn{7}{|l|}{ Number of prior therapies } \\
\hline \multicolumn{7}{|l|}{ I prior therapy } \\
\hline ORR, \% & 64.7 & 22.4 & $<0.00$ I & 66.1 & 29.8 & $<0.001$ \\
\hline Median TTP, months & NR & 5.1 & $<0.001$ & NR & 4.7 & $<0.001$ \\
\hline \multicolumn{7}{|l|}{$\geq 2$ prior therapies } \\
\hline ORR, \% & 58.7 & 18.3 & $<0.00$ I & 57.5 & 21.2 & $<0.001$ \\
\hline Median TTP, months & 10.2 & 4.6 & $<0.00 \mathrm{I}$ & 11.1 & 4.7 & $<0.001$ \\
\hline \multicolumn{7}{|l|}{ Prior therapies } \\
\hline \multicolumn{7}{|l|}{ Prior thalidomide } \\
\hline ORR, \% & 56.8 & 12.5 & $<0.00 \mathrm{I}$ & 49.1 & 16.4 & 0.002 \\
\hline Median TTP, months & 8.5 & 4.1 & $<0.00$ I & 8.4 & 4.6 & $<0.001$ \\
\hline \multicolumn{7}{|l|}{ No prior thalidomide } \\
\hline ORR, \% & 64.1 & 26.0 & $<0.001$ & 65.0 & 28.7 & $<0.001$ \\
\hline \multicolumn{7}{|l|}{ Prior bortezomib } \\
\hline ORR, \% & 68.4 & 10.0 & $<0.00$ I & & & \\
\hline Median TTP, months & 10.3 & 3.3 & $<0.00$ I & & & \\
\hline \multicolumn{7}{|l|}{ No prior bortezomib } \\
\hline ORR, \% & 60.1 & 21.2 & $<0.00$ I & & & \\
\hline \multicolumn{7}{|l|}{$\beta_{2}$-microglobulin level } \\
\hline \multicolumn{7}{|l|}{$<2.5 \mathrm{mg} / \mathrm{L}$} \\
\hline ORR, \% & 75.0 & 27.5 & $<0.001$ & 70.6 & 37.5 & $<0.001$ \\
\hline \multicolumn{7}{|l|}{$\geq 2.5 \mathrm{mg} / \mathrm{L}$} \\
\hline ORR, \% & 55.2 & 16.8 & $<0.001$ & 56.0 & 18.9 & $<0.001$ \\
\hline \multicolumn{7}{|l|}{ Prior SCT } \\
\hline \multicolumn{7}{|l|}{ Yes } \\
\hline ORR, \% & 66.1 & 19.4 & $<0.001$ & 61.9 & 28.4 & $<0.001$ \\
\hline \multicolumn{7}{|l|}{ No } \\
\hline ORR, \% & 52.9 & 20.6 & $<0.001$ & 58.2 & 18.8 & $<0.001$ \\
\hline
\end{tabular}

Abbreviations: Dex, placebo plus dexamethasone; Leni + Dex, lenalidomide plus dexamethasone; NR, not reached; ORR, overall response rate; SCT, stem cell transplantation; TTP, time to progression.

thalidomide $(64 \%$ vs $28 \% ; P<0.01) .{ }^{99}$ When patients who had received prior thalidomide were divided into three subgroups based on the degree of thalidomide resistance, the ORR was similar across resistance groups. Even the group with the strongest resistance to thalidomide (ie, never responded nor had stable disease) had a higher response rate $(P<0.01)$.

In another prospective subgroup analysis, the benefits of starting lenalidomide therapy at first relapse were assessed by comparing outcomes with lenalidomide plus dexamethasone versus dexamethasone alone among patients who had received one versus $\geq 2$ prior therapies. ${ }^{100}$ Among the 248 of 692 patients who had received only one prior therapy, those assigned to second-line lenalidomide plus dexamethasone had a significantly higher ORR than those receiving dexamethasone alone (65\% vs $26 \%$, respectively). Among the 456 patients who had received $\geq 2$ prior therapies, those treated with lenalidomide plus dexamethasone also had a significantly higher ORR than those treated with dexamethasone alone (58\% vs $20 \%$, respectively). Comparing patients who received lenalidomide plus dexamethasone as second-line versus later salvage therapy, the ORR appeared higher with early treatment. A higher proportion of patients receiving second-line therapy had previously had SCT (66\% vs 54\%), whereas more patients receiving later salvage therapy had previously received thalidomide $(53.2 \%$ vs $12.5 \%)$ and bortezomib (11.6\% vs $0.4 \%)$. 
In further subanalyses of MM-009 and MM-010, Foa and colleagues reported that among 154 patients with IgA disease at baseline, lenalidomide plus dexamethasone was associated with a significantly higher ORR than dexamethasone alone ( $68.1 \%$ vs $18.3 \%$, respectively; $P<0.001) .{ }^{101}$ The CR rate in patients with IgA disease who were treated with lenalidomide plus dexamethasone, versus dexamethasone alone, was $18.1 \%$ and $0 \%$, respectively $(P=n s)$. Similarly, in patients without IgA disease at baseline, lenalidomide plus dexamethasone achieved a higher ORR compared with dexamethasone alone $(57.7 \%$ vs $23.0 \%$, respectively; $P<0.001)$. A separate analysis demonstrated that the superiority of lenalidomide plus dexamethasone compared with dexamethasone alone was independent of baseline ECOG performance status. ${ }^{102}$ In this analysis, patients with an ECOG scores of 0 or $\geq 1$ had significantly higher ORR with lenalidomide plus dexamethasone (59\% and $62 \%$, respectively) compared with dexamethasone alone (22\% and $22 \%$, respectively; $P<0.001$ for both). Also, age did not determine response to lenalidomide, with another subanalysis showing that ORR was significantly higher for lenalidomide plus dexamethasone compared with dexamethasone alone for patients aged $<65$ years (61.5\% vs $22.2 \%$, respectively), $65-75$ years $(58.4 \%$ vs $21.4 \%$ ), and $>75$ years $(63.9 \%$ vs $29.9 \%){ }^{103}$

In a pooled subgroup analysis of 682 patients with serum creatinine levels of $\leq 2.5 \mathrm{mg} / \mathrm{dL}$ at baseline, lenalidomide plus dexamethasone significantly improved response rate compared with dexamethasone alone in patients with normal renal function (creatinine clearance $\left[\mathrm{Cr}_{\mathrm{Cl}}\right]>80 \mathrm{~mL} / \mathrm{min}$ : $63.9 \%$ vs $27.0 \%$, respectively; $P<0.001$ ) and in those with mild $\left(\mathrm{Cr}_{\mathrm{Cl}} \geq 50 \mathrm{~mL} / \mathrm{min}\right.$ to $<80 \mathrm{~mL} / \mathrm{min}: 64.0 \%$ vs $19.8 \%$; $P<0.001)$ and moderate $\left(\mathrm{Cr}_{\mathrm{Cl}} \geq 30 \mathrm{~mL} / \mathrm{min}\right.$ to $<50 \mathrm{~mL} / \mathrm{min}$ : $61.9 \%$ vs $20.6 \% ; P=0.001$ ) renal impairment ${ }^{104}$ (Table 5). The ORR was not significantly different between lenalidomide plus dexamethasone and dexamethasone alone in the 28 patients with severe renal impairment $\left(\mathrm{Cr}_{\mathrm{Cl}}<30 \mathrm{~mL} / \mathrm{min}\right.$ : $50.0 \%$ vs $25.0 \%$, respectively; $P=0.205$ ), with $\mathrm{CR}$ rates following a similar trend to ORR.

Finally, a post-hoc analysis of data from the MM-009 and MM-010 trials indicated that dexamethasone dose reductions improved the efficacy of lenalidomide plus dexamethasone treatment compared with patients who continued to receive dexamethasone at the planned dose. ${ }^{105}$ Patients assigned to lenalidomide plus dexamethasone and who had a subsequent dexamethasone dose reduction experienced a significantly higher ORR and CR rate (69.6\% and $23.9 \%$, respectively) compared with patients who continued to receive the standard
Table 5 Treatment response, time-to-progression and overall survival in MM-009 and MM-0I0: pooled subgroup analysis according to baseline renal impairment ${ }^{104}$

\begin{tabular}{|c|c|c|c|}
\hline & \multicolumn{3}{|c|}{ MM-009 and MM-0 I0 $(N=682)$} \\
\hline & Leni + Dex & Dex & $P$ value \\
\hline \multicolumn{4}{|l|}{$\mathrm{CR}_{\mathrm{Cl}}>80 \mathrm{~mL} / \mathrm{min}$} \\
\hline ORR, \% & 63.9 & 27.0 & $<0.001$ \\
\hline $\mathrm{CR}, \%$ & 16.5 & 1.8 & \\
\hline Median TTP, months & 11.3 & 4.7 & $<0.001$ \\
\hline Median OS, months & NR & 101.2 & 0.142 \\
\hline \multicolumn{4}{|c|}{$\mathrm{CR}_{\mathrm{Cl}} \geq 50$ to $<80 \mathrm{~mL} / \mathrm{min}$} \\
\hline ORR, \% & 64.0 & 19.8 & $<0.001$ \\
\hline $\mathrm{CR}, \%$ & 12.8 & 2.3 & \\
\hline Median TTP, months & 12.1 & 4.7 & $<0.001$ \\
\hline Median OS, months & 34.7 & 27.2 & 0.131 \\
\hline \multicolumn{4}{|c|}{$\mathrm{CR}_{\mathrm{CI}} \geq 30$ to $<50 \mathrm{~mL} / \mathrm{min}$} \\
\hline ORR, \% & 61.9 & 20.6 & 0.001 \\
\hline $\mathrm{CR}, \%$ & 21.4 & 0 & \\
\hline Median TTP, months & $1 \mathrm{I} .4$ & 2.8 & $<0.001$ \\
\hline Median OS, months & 30.4 & 12.5 & 0.068 \\
\hline \multicolumn{4}{|l|}{$\mathrm{CR}_{\mathrm{Cl}}<30 \mathrm{~mL} / \mathrm{min}$} \\
\hline ORR, \% & 50.0 & 25.0 & 0.205 \\
\hline $\mathrm{CR}, \%$ & 6.3 & 8.3 & \\
\hline Median TTP, months & 7.9 & 4.7 & 0.031 \\
\hline Median OS, months & 18.6 & 16.9 & 0.849 \\
\hline
\end{tabular}

Abbreviations: $\mathrm{CR}$, complete response; $\mathrm{CR}_{\mathrm{Cl}}$, creatinine clearance; Dex, placebo plus dexamethasone; Leni + Dex, lenalidomide plus dexamethasone; NR, not reached; ORR, overall response rate.

dexamethasone regimen in combination with lenalidomide (50.8\% and $13.0 \%$, respectively; $P<0.05$ for both).

In an ongoing Dutch compassionate need program, patients with relapsed or refractory MM were treated with lenalidomide $25 \mathrm{mg}$ /day on days 1-21 every 28 days, in combination with dexamethasone $40 \mathrm{mg} /$ day on days $1-4$ and 15-18 until disease progression, unacceptable toxicity, or for a maximum of eight courses. Fifteen patients received lenalidomide $10 \mathrm{mg} /$ day maintenance therapy without dexamethasone after 6-8 courses of therapy. ${ }^{106}$ The preliminary response data of the first 42 patients showed an ORR of $83 \%$ (CR 5\%, VGPR 45\%, PR 45\%, and MR 5\%).

\section{Single-agent lenalidomide}

In a multicenter, open-label phase II study of single-agent lenalidomide in relapsed or refractory MM, 102 patients were treated with either lenalidomide $30 \mathrm{mg}$ once daily or $15 \mathrm{mg}$ twice daily for 21 days of every 28 -day cycle. ${ }^{107}$ A total of $56 \%$ of patients had received at least four prior lines of 
therapy, 61\% had received prior high-dose chemotherapy followed by SCT, 76\% had received prior thalidomide, and $18 \%$ had previously received bortezomib. In the entire cohort, the ORR to lenalidomide was 25\% (24\% for once daily and $29 \%$ for twice daily), and a further $29 \%$ of patients responded with the addition of low-dose dexamethasone, which was permitted after two cycles for progressive or stable disease. The median duration of response, with censoring at the time that dexamethasone was added, was 19 months (range 2-22 months). In the twice-daily group, the median duration of response was 23 months (2-25 months). In a long-term follow-up of 15 patients who remained on therapy for a median of 4.1 years, 11 had achieved either CR or PR and continued to respond, including four of six patients receiving lenalidomide monotherapy (including a patient who progressed after 3.7 years), and seven of nine patients receiving concomitant dexamethasone. ${ }^{108}$ The remaining four patients maintained stable disease during this long-term follow-up.

A second multicenter, open-label study evaluated singleagent lenalidomide in 222 patients with relapsed or refractory MM (MM-014). ${ }^{111,112}$ Lenalidomide was administered at $30 \mathrm{mg}$ once daily on days $1-21$ every 28 days until disease progression or intolerance. Concomitant dexamethasone was not permitted. All patients had received at least two prior therapies, including bortezomib (43\%), thalidomide (80\%), and stem cell transplantation (45\%). The ORR was $26 \%$, with an additional $66 \%$ of patients achieving stable disease. The median duration of response was 13 months.

In a phase I dose-escalation study of 27 patients who received lenalidomide as a single daily dose, 24 patients received at least 28 days of therapy and were considered evaluable for response. ${ }^{113}$ Seventeen patients (71\%) had a best response of $\geq 25 \%$ reduction in M-protein, including seven patients (29\%) who achieved $\geq 50 \%$ reduction. The median duration of response was six months and the median time to response was two months.

\section{Lenalidomide plus doxorubicin}

In the relapsed or refractory MM setting, lenalidomide has been investigated in a phase I/II study in combination with pegylated liposomal doxorubicin-based chemotherapy. ${ }^{114}$ Sixty-two patients (median age 62 years) received liposomal doxorubicin $40 \mathrm{mg} / \mathrm{m}^{2}$ and vincristine $2 \mathrm{mg}$ on day 1 , dexamethasone $40 \mathrm{mg}$ /day on days $1-4$, and lenalidomide $5-15 \mathrm{mg} /$ day on days $1-21$ of every 28 -day cycle. Among 52 evaluable patients, the ORR of the combination was $75 \%$, including $29 \%$ of patients with either a CR or nCR.
Best response occurred after a median of 115 days and four cycles of therapy.

\section{Lenalidomide/cyclophosphamide/dexamethasone}

In a retrospective analysis of 21 patients who were administered lenalidomide $25 \mathrm{mg}$ /day on days 1-21, cyclophosphamide $500 \mathrm{mg} /$ day on days $1,8,15$, and 21 , and dexamethasone $40 \mathrm{mg}$ /day on days $1-4$ and $12-15$ of every 28 -day cycle for a maximum of nine cycles, 15 of 20 (75\%) evaluable patients had a response, including one CR, three VGPR, and nine PR. ${ }^{115}$ The median time to response was 31 days. There was no difference in response rate between patients who required a dose reduction compared with those who tolerated the full treatment schedule.

\section{Lenalidomide/doxorubicin/dexamethasone (RAD)}

In a phase I/II study, lenalidomide was evaluated in combination with doxorubicin and dexamethasone. ${ }^{116,117}$ A total of 69 patients (median age 65 years) received six 28-day cycles of lenalidomide $10-25 \mathrm{mg} /$ day on days $1-21$, doxorubicin 4-9 $\mathrm{mg} / \mathrm{m}^{2}$ as a 24-hour infusion on days $1-4$, and dexamethasone $40 \mathrm{mg}$ /day on days $1-4$ and 17-20, including 20 patients who received treatment at five lenalidomide and doxorubicin dose levels during phase I. In phase II of the study, all patients received the fifth dose level of lenalidomide $25 \mathrm{mg}$ on days $1-21$, doxorubicin $9 \mathrm{mg} / \mathrm{m}^{2}$ on days $1-4$, and dexamethasone $40 \mathrm{mg}$ on days $1-4$ and $17-20 .{ }^{117}$ G-CSF support was given at $6 \mathrm{mg}$ on day 6 . ORR for patients receiving treatment at dose levels $1-4$ in the phase I study was $60 \%$, including five patients $(25 \%)$ with nCR. ORR for the 41 patients receiving the highest dose level in phase II of the study was $85 \%$, including 10 patients (24\%) with CR and 24 patients $(59 \%)$ with VGPR.

\section{Lenalidomide plus prednisone}

In a study of 69 patients who received lenalidomide plus corticosteroids (pulsed dexamethasone or prednisone) as part of an Expanded Access Program in Canada, the ORR was $58 \%$ in patients aged $\geq 65$ years and older, and $56 \%$ in patients aged $<65$ years. ${ }^{118}$

\section{Lenalidomide plus bortezomib}

In the relapsed or refractory disease setting, the combination of lenalidomide and bortezomib in a phase I dose-escalation study of 36 patients yielded an ORR of $58 \%$, including $6 \%$ with CR or nCR. ${ }^{110}$ Lenalidomide was administered at a dose of $5,10,15$, or $20 \mathrm{mg}$ on days $1-14$, and bortezomib was given at either 1.0 or $1.3 \mathrm{mg} / \mathrm{m}^{2}$ on days $1,4,8$, and 11 of every 21-day cycle for a median of six cycles. The median duration 
of response was six months, with 11 patients remaining on therapy beyond one year. Dexamethasone was added in 14 patients with progressive disease, with an objective response subsequently achieved in 10 patients.

\section{RVd}

Lenalidomide may sensitize MM cells to bortezomib and dexamethasone, suggesting combination therapy may enhance clinical activity. In a recently completed phase II trial of 65 patients, 43 patients (median age 67 years) with relapsed or refractory MM have to date received up to eight cycles of lenalidomide $15 \mathrm{mg}$ on days 1-14 of a 21 -day cycle, bortezomib $1.0 \mathrm{mg} / \mathrm{m}^{2}$ on days $1,4,8$, and 11 of a 21-day cycle, and dexamethasone $40 \mathrm{mg}$ (cycles 1-4) or $20 \mathrm{mg}$ (cycles 5-8) twice weekly for two weeks of every 21-day cycle. ${ }^{62,119}$ Based on safety data, dexamethasone dosing was subsequently reduced to $20 \mathrm{mg}$ for cycles 1-4 and $10 \mathrm{mg}$ for cycles 5-8. In 33 evaluable patients with a median of two prior therapies including dexamethasone (90\%), thalidomide (78\%), and bortezomib (68\%), the ORR (minimal response or better) of major response or better was $73 \%$, including 36\% with CR, unconfirmed CR or VGPR. The median duration of response was 39 weeks. ${ }^{119}$

Terpos and colleagues compared lenalidomide $25 \mathrm{mg}$ /day on days $1-21$ every 28 days plus either high- $(n=38)$ or low-dose $(n=20)$ dexamethasone with the combination of lenalidomide $15 \mathrm{mg}$ /day on days $1-14$ every 21 days plus bortezomib $1.0 \mathrm{mg} / \mathrm{m}^{2}$ on days $1,4,8$, and 11 , and low-dose dexamethasone $(n=13) .{ }^{120}$ Currently, 50 patients have completed three cycles of therapy, including 38 of 58 patients assigned to lenalidomide plus either high- or low-dose dexamethasone and 12 of 13 patients assigned to $\mathrm{RVd}$. A total of 26 patients have received six cycles of therapy, including 19 of 58 patients assigned to lenalidomide and dexamethasone and seven of 13 patients assigned to $\mathrm{RVd}$. The ORR was 58\% in patients treated with lenalidomide and dexamethasone compared with $53 \%$ in patients treated with RVd.

\section{Bevacizumab/lenalidomide/dexamethasone (Bev/Rev/Dex)} In a phase II study, 17 patients received four-weekly cycles of lenalidomide $25 \mathrm{mg}$ /day on days 1-21, bevacizumab $10 \mathrm{mg} / \mathrm{kg}$ as a two-hour infusion every two weeks, and dexamethasone $40 \mathrm{mg}$ once a week. ${ }^{121}$ Among 10 evaluable patients who have completed at least four cycles of therapy, seven patients $(70 \%)$ achieved a PR after a median of two cycles and have maintained their response.
Lenalidomide/melphalan/prednisone/thalidomide (RMPT)

In a phase II study, 43 patients (median age 69 years) were administered six cycles of lenalidomide $10 \mathrm{mg}$ /day on days 1-21 every 28 days, melphalan $0.18 \mathrm{mg} / \mathrm{kg}$ on days $1-4$, prednisone $2 \mathrm{mg} / \mathrm{kg}$ on days $1-4$, and thalidomide $50-100$ $\mathrm{mg} /$ day on days 1-28 followed by maintenance therapy of lenalidomide $10 \mathrm{mg} /$ day. ${ }^{122}$ Therapy was administered as second-line in $61 \%$ of patients and third-line in 39\%. After two cycles, $52 \%$ of patients achieved at least PR and after a median of four cycles, 91\% achieved at least PR including $45 \%$ with VGPR.

\section{Time to progression}

\section{Lenalidomide plus dexamethasone}

In the MM-009 and MM-010 studies, TTP was the primary end point. The median TTP was significantly longer in patients assigned to lenalidomide plus dexamethasone compared with dexamethasone alone (MM-009: 11.1 months vs 4.7 months, respectively; $P<0.001$; MM-010: 11.3 months vs 4.7 months; $P<0.001)^{2,97}$ TTP was also significantly longer in the lenalidomide plus dexamethasone group compared with dexamethasone alone in patients who had received prior thalidomide or bortezomib therapy, and in patients with 1 or $\geq 2$ prior therapies (Table 4). In a pooled analysis of all 704 patients in both studies, the median TTP in patients treated with lenalidomide plus dexamethasone versus dexamethasone alone was 11.2 months versus 4.7 months $(P<0.001) .{ }^{97}$ Response was related to TTP as among the patients treated with lenalidomide plus dexamethasone who achieved a CR or nCR, median TTP was significantly longer than those who achieved a PR $(<15.1$ months vs 10.7 months; $P<0.001){ }^{98}$

Among the 154 patients with IgA disease at baseline, median TTP was significantly longer in the lenalidomide plus dexamethasone group than in the dexamethasone-only group (10.3 months vs 3.8 months, respectively; $P<0.001$ ). ${ }^{101}$ In patients without IgA disease, median TTP was again significantly longer in the lenalidomide plus dexamethasone group compared with dexamethasone alone (12.0 months vs 4.7 months, respectively; $P<0.001)$. Patients with a baseline ECOG score of 0 or $\geq 1$ also had a significantly longer median TTP on lenalidomide plus dexamethasone (10.3 months and 13.3 months, respectively) than dexamethasone alone (4.7 months and 4.7 months, respectively; $P<0.001$ for both comparisons). ${ }^{102}$ Dexamethasone dose reduction was similarly associated with a longer TTP. In the pooled subgroup analysis of patients with renal impairment, median TTP was significantly longer for lenalidomide plus dexamethasone 
compared with dexamethasone alone in patients with normal renal function (11.3 months vs 4.7 months, respectively; $P<0.001)$, and mild (12.1 months vs 4.7 months; $P<0.001$ ), moderate (11.4 months vs 2.8 months; $P<0.001$ ), and severe ( 7.9 months vs 4.7 months; $P=0.031$ ) renal impairment $^{104}$ (Table 5). In another subgroup analysis of patients who received lenalidomide plus dexamethasone, dose reduction of dexamethasone was associated with significantly longer TTP than continuing dexamethasone according to the planned dosing schedule $(>13.9$ months vs $>5.6$ months, respectively; $P=0.002) .{ }^{105}$

\section{Single-agent lenalidomide}

Among 222 patients enrolled in the multicenter, open-label phase II MM-014 study, 69\% of patients had disease progression by the end of the study with a median TTP of 5.4 months. ${ }^{112}$

\section{RAD}

In a phase I/II study of 41 patients treated for six 28-day cycles with lenalidomide $25 \mathrm{mg}$ /day on days 1-21, doxorubicin $9 \mathrm{mg} / \mathrm{m}^{2}$ on days $1-4$, dexamethasone $40 \mathrm{mg}$ /day on days $1-4$ and 17-20, and G-CSF 6 mg on day 6, median TTP after a median follow-up of five months was 9.3 weeks. ${ }^{117}$

\section{Overall survival}

\section{Lenalidomide plus dexamethasone}

Relative to dexamethasone alone, median OS was significantly prolonged in patients assigned to lenalidomide plus dexamethasone in both the MM-009 and MM-010 studies. ${ }^{2,3}$ At a median follow-up post-randomization of 17.1 months in the MM-009 study, the median OS in patients assigned to lenalidomide plus dexamethasone was 29.6 months versus 20.2 months for dexamethasone alone $(P<0.001) .{ }^{3}$ Similarly, at a median follow-up of 16.5 months in the MM-010 study, the median OS in patients assigned to lenalidomide plus dexamethasone had not been reached, whereas in patients assigned to dexamethasone-only median OS was estimated at 20.6 months $(P=0.03){ }^{2}$ With an extended follow-up of 31.3 months, the median OS for all 704 patients pooled from both studies was 35.0 months for those receiving lenalidomide plus dexamethasone and 31.0 months for those on dexamethasone alone $(P<<0.05) .{ }^{97}$ It should be noted that this significant difference in OS was maintained despite $47 \%$ of patients receiving dexamethasone alone crossing over to lenalidomide plus dexamethasone therapy. ${ }^{97}$ Response was correlated with survival because among patients assigned to lenalidomide plus dexamethasone the median OS was significantly higher for those who achieved CR or
nCR, than for patients who achieved a PR $(>30.9$ months vs $>27.5$ months, respectively; $P<0.01) .{ }^{98}$

In both MM-009 and MM-010, OS was significantly improved in the lenalidomide plus dexamethasone group compared with the dexamethasone-only group, among patients who had previously been treated with thalidomide., ${ }^{2,3}$ In addition, lenalidomide plus dexamethasone was associated with significantly longer OS compared with dexamethasone alone, irrespective of the number of prior therapies. ${ }^{97,123}$ In a pooled analysis of all 704 patients, the median OS was not yet reached in patients assigned to the lenalidomide plus dexamethasone group who had received one prior therapy compared with 35.3 months in patients assigned to dexamethasone alone $(P=0.24) .{ }^{97}$ In patients who had received $>1$ prior therapy, median OS was 32.4 months in those assigned to lenalidomide plus dexamethasone compared with 27.3 months in those assigned to dexamethasone alone $(P<0.05)$.

In patients with IgA disease at baseline, there was a trend towards improved OS with lenalidomide plus dexamethasone treatment compared with dexamethasone alone (30.3 months vs 23.8 months, respectively; $P=$ not significant). ${ }^{101}$ In patients without IgA disease at baseline, there was a significant benefit in terms of OS for lenalidomide plus dexamethasone versus dexamethasone alone (36.3 months vs 31.7 months, respectively; $P<0.05)$. Similarly, patients with an ECOG performance status of 0 at baseline had a similar median OS with lenalidomide plus dexamethasone relative to dexamethasone alone (36.3 months vs 37.0 months, respectively; $P=$ not significant). ${ }^{102}$ However, among patients with an ECOG score $\geq 1$, median OS was significantly higher in patients assigned to lenalidomide plus dexamethasone versus dexamethasone alone (32.9 months vs 24.1 months, respectively; $P<0.01$ ). When patients were stratified according to renal function, there was a trend towards improved OS with lenalidomide plus dexamethasone compared with dexamethasone alone in patients with moderate renal impairment (30.4 months vs 12.5 months, respectively; $P=0.068)^{104}$ (Table 5). However, OS was not significantly different for those with normal renal function (not reached vs 101.2 months, respectively; $P=0.142$ ), mild renal impairment (34.7 months vs 27.2 months; $P=0.131$ ) or severe renal impairment (18.6 months vs 16.9 months; $P=0.849$ ). Among patients who were assigned to lenalidomide plus dexamethasone, dose reduction of dexamethasone was associated with a trend towards improved OS compared with patients who were maintained on the planned dexamethasone dose regimen ( $>28.3$ months vs $>25.5$ months, respectively; $P=0.19) .{ }^{105}$ 
In the MM-009/ and MM-010 studies, $47 \%$ of patients randomized to dexamethasone alone later switched to lenalidomide plus dexamethasone at disease progression or following ethical study unblinding. ${ }^{24}$ In a survival analysis that adjusted for the overestimation of survival in the group treated with dexamethasone alone, Morgan and colleagues reported that treatment of patients who had one prior therapy with single-agent dexamethasone yielded a median survival of 16.2 months compared with 33.6 months following crossover to lenalidomide plus dexamethasone. ${ }^{124}$ The median survival for patients with multiple prior therapies was 12.6 months compared with 27.3 months with crossover to lenalidomide plus dexamethasone. Using a lifetime simulation model, Morgan and colleagues estimated a mean survival of 2.2 life-years with dexamethasone alone compared with 5.6 lifeyears with lenalidomide plus dexamethasone for patients with one prior therapy. For patients with multiple prior therapies, lifetime simulation yielded an estimated mean survival of 1.5 life-years for dexamethasone alone compared with 4.2 life-years for lenalidomide plus dexamethasone.

The MM-016 study was a multicenter, single-arm, open-label expanded access program for lenalidomide in relapsed and refractory MM that reported on the efficacy of lenalidomide plus dexamethasone in patients according to their del13q, t( $4 ; 14)$, and del17p13 status. Patients received lenalidomide $25 \mathrm{mg}$ /day on days 1-21 of a 28-day cycle, plus dexamethasone $40 \mathrm{mg} /$ day on days 1-4, 9-12 and 17-20 for four cycles, then days $1-4$ only beginning with cycle $5 .{ }^{125}$ In the entire group, progression-free survival (PFS) was 10.6 months and the median OS was not reached at a median follow-up of 16 months. Compared with the overall cohort, treatment with lenalidomide plus dexamethasone overcame poor prognosis conferred by del13q and $t(4 ; 14)$ cytogenetic abnormalities, with no increased risk of a reduction in OS (del13q: hazard ratio [HR], 0.56, 95\% confidence interval [CI] 0.25-1.29; $P=0.179$; and t( $4 ; 14)$ : HR, $1.26,95 \% \mathrm{CI}$ $0.46-3.42 ; P=0.641)$. However, patients with del17p13 had a reduced $\mathrm{OS}$ despite a rapid initial response to therapy (HR, 3.83 ; 95\% CI 1.34-10.93; $P=0.012$ ).

In a preliminary analysis of 42 patients with relapsed or refractory MM treated with lenalidomide and dexamethasone in an ongoing Dutch compassionate need program, the median OS has not been reached (median PFS 10 months). ${ }^{106}$

\section{Single-agent lenalidomide}

In an open-label, phase II study of 102 patients, at a median follow-up of 31 months, lenalidomide $30 \mathrm{mg}$ /day was associated with a median OS of 27 months. There was no significant survival advantage reported for patients who received $30 \mathrm{mg}$ once-daily dosing versus $15 \mathrm{mg}$ twice daily. ${ }^{107}$ In the multicenter, open-label, phase II MM-014 study of 222 patients, in which concomitant dexamethasone was not permitted, three-year OS was $41 \%$, with a median OS of 1.9 years. ${ }^{112}$

\section{Lenalidomide and bortezomib}

Overall survival in the lenalidomide plus bortezomib is emerging at 37 months.

\section{Lenalidomide plus prednisone}

Among 69 patients who received lenalidomide plus corticosteroids (pulsed dexamethasone or prednisone) as part of an Expanded Access Program in Canada, OS was $74 \%$ in patients aged $\geq 65$ years compared with $76 \%$ in patients $<65$ years. ${ }^{118}$

\section{RAD}

In a phase I/II study of 41 patients treated for six 28-day cycles with lenalidomide $25 \mathrm{mg}$ /day on days 1-21, doxorubicin $9 \mathrm{mg} / \mathrm{m}^{2}$ on days $1-4$, dexamethasone $40 \mathrm{mg} /$ day on days 1-4 and 17-20, and G-CSF $6 \mathrm{mg}$ on day 6, after a median follow-up of five months OS was 79\%. ${ }^{117}$

\section{Safety and tolerability}

In the two pivotal phase III studies of relapsed or refractory $\mathrm{MM}$, grade 3 or 4 adverse events were reported more frequently in patients assigned to lenalidomide plus dexamethasone compared with dexamethasone alone. ${ }^{2,3}$ In the MM-009 study, grade 3 or 4 hematologic adverse events in the lenalidomide plus dexamethasone versus dexamethasone-only groups were neutropenia ( $41.2 \%$ vs $4.5 \%$, respectively), anemia ( $13.0 \%$ vs $5.1 \%$ ), thrombocytopenia ( $14.7 \%$ vs $6.9 \%$ ), and febrile neutropenia ( $3.4 \%$ vs $0 \%$ ). Other commonly occurring grade 3 or 4 adverse events were any infection $(21.4 \%$ vs $12.0 \%$, respectively), pneumonia ( $12.4 \%$ vs $7.4 \%)$, hyperglycemia ( $10.8 \%$ vs $8.6 \%)$, hypokalemia ( $6.2 \%$ vs $1.1 \%)$, and fatigue (6.2\% vs $6.3 \%)$. VTE events occurred in $14.7 \%$ of patients in the lenalidomide plus dexamethasone group compared with $3.4 \%$ of patients in the dexamethasone-only group $(P<0.001) .{ }^{3}$ In the MM-010 study, grade 3 or 4 hematologic adverse events in the lenalidomide plus dexamethasone versus dexamethasone-only groups were neutropenia $(29.5 \%$ vs $2.3 \%$, respectively), anemia ( $8.6 \%$ vs $6.9 \%$ ), thrombocytopenia ( $11.4 \%$ vs $5.7 \%$ ), and febrile neutropenia (3.4\% vs $0 \%$ ). Other commonly occurring grade 3 or 4 adverse events were any infection ( $11.3 \%$ vs $6.2 \%$, respectively), muscle weakness $(7.4 \%$ vs $4.6 \%)$, asthenia $(6.2 \%$ vs $5.7 \%)$, and fatigue ( $6.8 \%$ vs $3.4 \%)$. Grade 3 or 4 VTE events occurred 
in $11.4 \%$ of patients in the lenalidomide plus dexamethasone group compared with $4.6 \%$ of patients in the dexamethasoneonly group. ${ }^{2}$

The increased incidence of VTE in patients receiving lenalidomide plus dexamethasone compared with dexamethasone alone does not appear to affect survival. In an analysis of 177 patients assigned to receive lenalidomide plus dexamethasone in the MM-009 study, OS $(P=0.4)$ and TTP $(P=0.7)$ were not significantly different for the 31 patients who experienced DVT compared with patients who did not experience DVT. ${ }^{126}$ In the MM-009 and MM-010 studies, multivariate analysis indicated that lenalidomide plus dexamethasone treatment with adjunctive erythropoietin was independently correlated with thrombosis; older age, lower plasma cell involvement in the bone marrow, and better ECOG performance status had a weaker association with thrombosis. ${ }^{127}$ None of the 23 patients who used aspirin during the first month of treatment developed thromboses; all events occurred in patients with rising M-protein levels at baseline.

In the MM-009 and MM-010 studies, the predominant reason for adjusting dexamethasone dose among patients assigned to lenalidomide plus dexamethasone was for an adverse event (41 of 46 patients). ${ }^{105}$ In this group of patients, reducing dexamethasone dose yielded a similar safety profile to those who did not require dose reductions. Grade 3 or 4 hematological events in patients who received dexamethasone dose reductions relative to those who maintained the planned dexamethasone dose were: neutropenia $(23.7 \%$ vs $32.6 \%$, respectively), thrombocytopenia ( $8.5 \%$ vs $6.8 \%$ ), and anemia $(6.8 \%$ vs $6.2 \%)$.

Among 1,400 patients with relapsed or refractory MM who were administered lenalidomide $25 \mathrm{mg}$ plus high-dose dexamethasone in 28-day cycles as part of an expanded access program in North America, the most commonly reported grade 3 or 4 adverse events were: neutropenia $(7.9 \%)$, thrombocytopenia $(6.0 \%)$, fatigue $(3.6 \%)$, anemia (3.5\%), pneumonia (3.1\%), and hyperglycemia $(2.0 \%){ }^{128}$ Although the grade 3 or 4 adverse events were the same as those reported in the two phase III studies, their frequencies were lower. Likewise, the most commonly reported adverse events of all grades were the same as those reported in the two pivotal studies.

The findings of a recent analysis of 72 patients receiving lenalidomide plus dexamethasone as first-line therapy indicate that myelosuppression is associated with renal dysfunction. ${ }^{129}$ In this analysis, eight of 14 patients with grade 3 or 4 myelosuppression had a baseline $\mathrm{Cr}_{\mathrm{Cl}}$ of $\leq 40 \mathrm{~mL} / \mathrm{min}$, with Kaplan-Meier analysis showing a significant association between renal insufficiency and time to myelosuppression. In the subgroup analysis of patients in the MM-009 and MM-010 studies, patients with renal impairment at baseline tended to have an increased incidence of thrombocytopenia compared with those with normal renal function. ${ }^{104}$ In patients treated with lenalidomide plus dexamethasone, the incidences of neutropenia and thrombocytopenia increased among those with normal renal function from $31.0 \%$ and $7.0 \%$, respectively, to $39.2 \%$ and $16.0 \%$ for mild renal impairment, and to $42.9 \%$ and $19.0 \%$ for moderate renal impairment, respectively. The incidences of neutropenia and thrombocytopenia among the 16 patients with severe renal impairment were $37.5 \%$ and $37.5 \%$, respectively. In the dexamethasoneonly arm, the incidences of neutropenia and thrombocytopenia among those with normal renal function were $4.3 \%$ and $5.5 \%$, respectively $(P<0.001$ and $P=0.649$ relative to the lenalidomide plus dexamethasone arm), compared with $1.5 \%$ and $5.3 \%$ in patients with mild renal impairment $(P<0.001$ and $P=0.007), 5.9 \%$ and $17.6 \%$ for moderate renal impairment $(P<0.001$ and $P=1.00)$, and $8.3 \%$ and $>0 \%$ in the 12 patients with severe renal impairment $(P=0.184$ and $P=0.024)$, respectively. There were no significant differences in the incidences of thrombotic episodes in lenalidomide plus dexamethasone versus dexamethasone-only patients with mild ( $12.0 \%$ vs $6.1 \%$, respectively; $P=0.126)$, moderate (14.3\% vs $2.9 \% ; P=0.122)$, or severe $(6.3 \%$ vs $8.3 \%$; $P=1.00)$ renal impairment.

In a pooled analysis of the MM-009 and MM-010 studies, the incidence of diarrhea was $39 \%$ in the lenalidomide plus dexamethasone arm compared with $28 \%$ in the dexamethasoneonly arm. ${ }^{130}$ Multivariate analysis found that therapy duration but not treatment assignment predicted diarrhea. Among a cohort of patients who received 9-15 months of therapy, the incidence of diarrhea after adjustment for treatment duration was similar for lenalidomide plus dexamethasone versus dexamethasone alone ( $42.3 \%$ vs $42.5 \%$, respectively), suggesting that the risk of unexpected diarrhea with long-term therapy may be partly attributable to dexamethasone, but it is important to note that mild-to-moderate diarrhea is a well recognized effect of lenalidomide monotherapy, particularly with prolonged use.

As a single-agent therapy in the relapsed or refractory MM setting, lenalidomide is again associated with myelosuppression. In a phase I dose-escalation study of lenalidomide 5-50 mg/day, neutropenia was the most common adverse event, with grade 3 neutropenia occurring in 15 of $25(60 \%)$ patients and grade 4 neutropenia in four of $25(16 \%)$ patients. ${ }^{113}$ Grade 3 thrombocytopenia occurred 
in five of $25(20 \%)$ patients. In a phase II study evaluating lenalidomide $30 \mathrm{mg}$ once-daily versus $15 \mathrm{mg}$ twice-daily, an increased incidence of cytopenia was noted in the twice-daily group, prompting a once-daily schedule moving forward. ${ }^{107}$ In a long-term follow-up of 15 patients treated initially with either $30 \mathrm{mg}$ once daily $(\mathrm{n}=11)$ or $15 \mathrm{mg}$ twice daily $(\mathrm{n}=4)$, with or without the addition of dexamethasone, the most common grade 3 or 4 toxicity was neutropenia, which occurred in 10 patients. ${ }^{108}$ No grade 3 or 4 thrombocytopenia, anemia, peripheral neuropathy, or DVT was reported. In a subsequent phase II study of 222 patients with relapsed or refractory MM, the most frequent grade 3 or 4 toxicities with single-agent lenalidomide $30 \mathrm{mg}$ once daily given on days $1-21$ of every 28-day cycle were neutropenia (60\%), thrombocytopenia (39\%), and anemia (20\%). ${ }^{112}$ However, the incidence of DVT and febrile neutropenia was low (both 4\%).

Prior to receiving regulatory approval, both thalidomide and lenalidomide were associated with VTE incidences $>20 \%$ when combined with dexamethasone for use as an off-label treatment for MM. ${ }^{131}$ In a systematic review of VTE rates, a search of the US FDA's MedWatch program found reports of VTE among eight lenalidomide-treated cancer patients, including three receiving aspirin prophylaxis, two on warfarin, and one on low-molecular-weight heparins. ${ }^{131}$ Clinical trials identified VTE in 38 of 278 (13.7\%) previously untreated patients and 48 of 346 (13.9\%) relapsed or refractory MM patients receiving lenalidomide plus dexamethasone. None of these patients received routine thromboprophylaxis. In another systematic review, VTE rates ranged from $8.5 \%-75 \%$ in MM patients treated with lenalidomide and dexamethasone or erythropoietin. However, with the addition of aspirin this rate was $<3.4 \%$. ${ }^{132} \mathrm{NCCN}$ guidelines currently recommend anticoagulation therapy in patients treated with lenalidomide plus dexamethasone. ${ }^{27}$ However, controlled studies may be needed to identify optimal thromboprophylaxis for patients treated with lenalidomide and dexamethasone.

In combination with bortezomib $1.0 \mathrm{mg} / \mathrm{m}^{2}$ and dexamethasone $20 \mathrm{mg} / 10 \mathrm{mg}$, lenalidomide $15 \mathrm{mg}$ administered for up to eight cycles is associated with manageable toxicities consisting mainly of grade 1 or 2 myelosuppression. ${ }^{119}$ Attributable nonhematologic toxicities were DVT in two of 41 patients, grade 3 atrial fibrillation in two patients, and grade 3 peripheral neuropathy in one patient. Dose reductions were required for lenalidomide in nine patients, bortezomib in five patients, and dexamethasone in 14 patients.

The combination of lenalidomide $10 \mathrm{mg} /$ day with melphalan $0.18 \mathrm{mg} / \mathrm{kg}$, prednisone $2 \mathrm{mg} / \mathrm{kg}$ and thalidomide $50-100 \mathrm{mg}$ was generally well tolerated in patients who received up to six cycles of therapy as second- or third-line treatment. ${ }^{122}$ The most frequent adverse events were hematologic, with $48 \%$ of patients experiencing grade 3 neutropenia and 16\% experiencing grade 4 neutropenia. Grade 3 and 4 thrombocytopenia were reported in 26\% and $10 \%$ of patients, respectively. Growth factor support was required in $39 \%$ of patients and one 1 patient required platelet transfusion. The most frequent nonhematologic toxicity was infection in $19 \%$ of patients. No VTE events were detected.

In 41 patients treated with lenalidomide $25 \mathrm{mg}$ (days 1-21) in combination with doxorubicin $9 \mathrm{mg} / \mathrm{m}^{2}$ (days $1-4$ ), dexamethasone $40 \mathrm{mg}$ (days 1-4 and 17-20), and G-CSF $6 \mathrm{mg}$, grade 3 or 4 infection occurred in $10 \%$ of patients and VTE occurred in 5\%. ${ }^{117}$ Eight patients prematurely discontinued due to catheter-related septicemia $(n=2)$, thrombosis of basal artery $(n=1)$, prolonged pneumonia $(n=1)$, or withdrawal of consent $(n=4)$. Adverse events were generally of moderate severity and manageable.

\section{Ongoing clinical development}

The encouraging results of the two pivotal phase III studies demonstrating that lenalidomide in combination with dexamethasone significantly prolongs survival compared with dexamethasone alone, has led to further studies in previously treated MM patients. Among the phase III or IV studies currently being conducted in this setting, lenalidomide is being evaluated in combination with: dexamethasone; bortezomib and dexamethasone; and dexamethasone with or without thalidomide. Lenalidomide is additionally being evaluated as maintenance therapy following ASCT. Other investigational combinations currently being investigated in phase I and II trials include lenalidomide plus dexamethasone in combination with each of the following: panobinostat, bevacizumab, SGN-40, perifosine, vorinostat, dasatinib, NPI-0002, and carfilzomib. Lenalidomide is also being studied in combination with everolimus, and as monotherapy in patients who have relapsed on prior SCT.

The finding that lenalidomide in combination with dexamethasone yields high objective response and survival rates at one-, two-, and three-year follow-up has also encouraged further research in newly diagnosed MM. In this setting, lenalidomide is being evaluated in phase III studies as single-agent therapy (melphalan/prednisone/thalidomide and single-agent dexamethasone as comparators), and for use in combination with dexamethasone, and melphalan and prednisone in patients aged $>65$ years. There is now evidence that initial induction therapy with a lenalidomide-based regimen does not prevent harvest of adequate numbers of 
$\mathrm{CD} 4^{+}$positive stem cells for autologous SCT, but appears to be dependent on mobilization using a combination of G-CSF and cyclophosphamide, or similar. ${ }^{94-96}$ Numerous phase I and II studies are currently investigating lenalidomide combination regimens in previously untreated patients including lenalidomide and bortezomib plus dexamethasone, with or without cyclophosphamide, and lenalidomide and bortezomib plus dexamethasone and doxorubicin. Lenalidomide is additionally being evaluated as maintenance therapy following autologous SCT.

\section{Economic evidence and resource utilization}

Limited information on the health economics of lenalidomide in MM comes from a budget impact model comparing resource utilization of four approved therapies in the US. ${ }^{133}$ This study used a managed-care payer perspective to assess resource utilization in MM associated with each of single-agent bortezomib, bortezomib plus pegylated liposomal doxorubicin, thalidomide plus dexamethasone, and lenalidomide plus dexamethasone. Drug costs were calculated based on average wholesale price less $15 \%$, with a $10 \%$ patient coinsurance contribution for thalidomide plus dexamethasone and lenalidomide plus dexamethasone, and a $20 \%$ patient contribution for single-agent bortezomib and bortezomib plus doxorubicin. Costs of therapy and costs of treating adverse events were based on standard sources or from peer-reviewed publications and/or meeting presentations. Incidences of adverse events, and assumptions for supportive care and prophylaxis were obtained from the prescribing information for each of the approved therapies and from published reports of pivotal phase III trials. Duration of therapy was based on the published median duration of therapy.

In this model, total costs for each of the four regimens were primarily driven by direct drug costs, with an acquisition cost of US $\$ 64,806$ for the combination of lenalidomide plus dexamethasone. ${ }^{133}$ This represented a 1.7 -fold increase on drug costs for the thalidomide plus dexamethasone combination, and a 1.9-fold increase on drug costs for the bortezomib plus doxorubicin combination. However, associated medical costs of lenalidomide plus dexamethasone (US \$1,623) were comparable to thalidomide plus dexamethasone, and less than a quarter of that of bortezomib plus doxorubicin. Costs attributable to adverse events were again favorable for the lenalidomide plus dexamethasone combination (US \$5,243), representing a cost-saving of US $\$ 2,667$ compared with thalidomide plus dexamethasone, and US \$851 compared with bortezomib plus doxorubicin. The total cost of the lenalidomide plus dexamethasone regimen including cost of prophylaxis for DVT and pulmonary embolism was US \$72,822, which represents a 1.5-fold higher total cost compared with either thalidomide plus dexamethasone, or bortezomib plus doxorubicin.

This study has several weaknesses of which the most important is that it does not account for differences in efficacy as a function of cost. No consideration was given to the patient populations, which in the pivotal phase III trials of lenalidomide involved a heavily pretreated population with relapsed or refractory disease, with a consequent impact on duration of therapy and adverse events. As oral drugs, lenalidomide and thalidomide in combination with dexamethasone would be expected to offer an improvement in health-related quality of life compared with combination bortezomib plus doxorubicin, which are administered as intravenous infusions. However, this study offers a starting point for comparisons between MM therapies and is a valid approach to economic analysis from the viewpoint of the payer. Additional health economic studies of lenalidomide are required that include quality of life measures and data on the cost utility of treatment.

In a chart review conducted in five university hospitals in France during the period 2004-2007, the total direct costs of usual care of patients with relapsed or refractory MM were estimated at $€ 73,000$ per patient from first relapse until death or last follow-up. ${ }^{134}$ The study included a total of 102 patients with a mean age at diagnosis of 59 years and a mean of 2.8 lines of therapy since first relapse. Novel agents were used in 205 of 281 lines (73\%) and consisted of thalidomide combination therapy (28\%), bortezomib (22\%), lenalidomide (13\%), and bortezomib plus thalidomide (10\%). The average cost per line was $€ 26,510$ including $€ 17,525$ for drugs. With respect to the third-line of treatment, lenalidomide-based therapy was similar to bortezomib: mean duration and cost of treatment for lenalidomide was 7.4 months and $€ 46,724$ compared with 6.9 months and $€ 46,321$ for bortezomib.

Deniz and colleagues used a discrete event simulation model to estimate the long-term health and cost consequences of lenalidomide plus dexamethasone versus dexamethasone-alone in MM patients who received either 1 or $\geq 2$ prior therapies. ${ }^{135}$ The model used patient responses to treatment and time-to-event data based on Weibull functions derived from pooled data from the MM-009 and MM-010 clinical studies. Long-term results from UK Medical Research Council-sponsored trials and Mayo Clinic data were used to calculate dexamethasone 
survival given that $47 \%$ of patients in the dexamethasone arm of the MM-009 and MM-010 trials crossed over to receive lenalidomide treatment following disease progression or ethical unblinding. Disease management costs were reflective of clinical practice in Wales, UK. Cost and health outcomes were discounted at $3.5 \%$ per annum to adjust to present values. Events and costs were considered over two years to reflect trial follow-up, whereas survival and quality-adjusted life years (QALYs) were modeled to end of life to avoid truncation bias. In patients with one prior therapy, lenalidomide plus dexamethasone was associated with improvements in both survival and QALYs (4.54 projected mean life years and 3.20 QALYs) compared with dexamethasone alone (2.00 and 1.39, respectively). This equated to an incremental cost per life year gained of $£ 20,617$ and per QALY gained of $£ 28,943$ in patients receiving lenalidomide. Similarly, in patients with at least two prior therapies, lenalidomide plus dexamethasone was associated with a projected mean survival of 3.61 life years and 2.50 QALYs compared with 1.41 life years and 1.00 QALYs for dexamethasone alone. The incremental cost of lenalidomide per life year gained in this group of patients was $£ 19,218$ and $£ 28,184$ per incremental QALY gained.

\section{Patient group/population}

The evidence to support lenalidomide in its licensed indication for use in combination with dexamethasone for the treatment of patients with relapsed or refractory MM who have undergone at least one prior therapy was predominantly derived from two pivotal phase III studies that compared lenalidomide plus high-dose dexamethasone with dexamethasone alone. ${ }^{2,3}$ MM-009 was conducted in 48 centers in the USA and Canada, and MM-010 was conducted in 51 centers in Europe, Australia, and Israel. The median age of patients was 63 years, most were male, and most had an ECOG performance status of 0 or 1 . Approximately $65 \%$ of patients had Durie-Salmon stage III disease at diagnosis, three-quarters of patients had lytic disease, and a third had bone marrow involvement. A total of $61 \%$ of patients enrolled in MM-009 and 55\% of patients in MM-010 had previously received at least one prior $\mathrm{SCT}$, and most had received $\geq 2$ previous lines of treatment. In MM-009, $10 \%$ of patients had previously received bortezomib, $44 \%$ had received thalidomide, and $60 \%$ had received dexamethasone. The respective data for patients enrolled in MM- 010 were $4 \%$, $34 \%$, and $67 \%$.

The patients enrolled in these studies represented a heavily pretreated population with advanced disease. A high proportion of patients $(40.5 \%)$ were aged $>65$ years. ${ }^{136}$ Of the elderly group, the median time to diagnosis was approximately 3.3 years, and three-quarters had received $\geq 2$ prior therapies, including dexamethasone in $69 \%$ and thalidomide in $32 \%$ of patients. However, the clinical benefit of lenalidomide plus dexamethasone in terms of response, TTP, and OS was comparable with younger patients.

Of the 353 patients in MM-009 and MM-010 who were randomized to receive lenalidomide plus dexamethasone, $210(59.5 \%)$ had previously undergone autologous SCT. ${ }^{137}$ In a subgroup analysis comparing outcomes in patients with prior autologous SCT and no prior autologous SCT, there were no significant differences in ORR ( $63 \%$ vs $55 \%$, respectively) or CR rate ( $13 \%$ vs $16 \% ; P=0.12)$. There was a trend towards prolonged TTP in patients without prior autologous SCT (14.2 vs 10.2 months for previous autologous SCT; $P=0.13)$. An interesting observation was that the median time from first pathologic diagnosis was similar for the two groups (3.4 years in the prior autologous SCT group vs 2.9 years in the no prior autologous SCT group). Based on the TTP trend, this observation implies that patients who have not had a chance to benefit from autologous SCT may receive an advantage from lenalidomide plus dexamethasone therapy, and provides a rationale for commencing lenalidomide-based therapy early in the disease course. ${ }^{137}$ This is further supported by the findings of a subgroup analysis, which suggested there was an advantage for second-line compared with later salvage treatment in terms of response rate and TTP. ${ }^{100}$

Thus, in patients with relapsed or refractory disease, the data indicate that treatment with lenalidomide plus dexamethasone is suitable as early or later salvage therapy in a broad group of patients. In particular, lenalidomide plus dexamethasone is effective at prolonging TTP independently of patient age, number or type of previous therapies including previous autologous SCT, and $\beta_{2}$-microglobulin status. ${ }^{2,3}$ Moreover, the combination of lenalidomide plus dexamethasone is effective at prolonging OS irrespective of prior thalidomide use or the number of previous therapies; OS is also improved in patients with IgA disease at baseline and in patients with an ECOG performance status $>0$.,3,101,102 In the relapsed or refractory setting, lenalidomide is emerging as a suitable partner for bortezomib, with nonoverlapping toxicities and a high rate of response. ${ }^{62,119}$

There is now increasing evidence to support a role for lenalidomide-based regimens as a first-line option where ORR $>90 \%$ have been reported, including CR rates of $18 \%-25 \% .{ }^{43,83,129}$ In newly diagnosed patients with ASIPs, the BiRD combination of lenalidomide, clarithromycin, 
and dexamethasone (BiRD) is associated with a $\mathrm{CR}$ rate of $71 \%$ and a VGPR or better rate of $96 \% .{ }^{88}$

\section{Dosage, administration, and formulation}

Lenalidomide (CC-5013, Revlimid ${ }^{\circledR}$ ), an immunomodulatory drug with antitumor, antiangiogenesis, and apoptotic activities, is an analog of thalidomide with more potent activity and a different tolerability profile. It is available for oral administration in $5 \mathrm{mg}, 10 \mathrm{mg}, 15 \mathrm{mg}$, and $25 \mathrm{mg}$ capsules. Lenalidomide is indicated in combination with dexamethasone for the treatment of patients with MM who have received at least one prior therapy. The recommended starting dose is $25 \mathrm{mg} /$ day with water, administered as a single $25 \mathrm{mg}$ capsule on days 1-21 of a repeated 28-day cycle. The recommended dose of dexamethasone is $40 \mathrm{mg}$ /day on days $1-4,9-12$, and 17-20 of each 28-day cycle for the first four cycles of therapy, and then at a dose of $40 \mathrm{mg} /$ day on days $1-4$ every 28 days. Dose modifications and interruptions are recommended to manage grade 3 or 4 neutropenia or thrombocytopenia, or other grade 3 or 4 lenalidomide-associated toxicities. ${ }^{138}$ In thrombocytopenia, when platelets fall to $<30,000$ per $\mu \mathrm{L}$, lenalidomide treatment should be interrupted and follow-up complete blood counts performed weekly until recovery is confirmed ( $\geq 30,000$ per $\mu \mathrm{L})$. Treatment should be restarted at $15 \mathrm{mg}$ /day. For each subsequent platelet fall to $<30,000$ per $\mu \mathrm{L}$, treatment should again be interrupted and resumed at $5 \mathrm{mg}$ less than the previous dose when platelet levels recover to 30,000 per $\mu \mathrm{L}$. In neutropenia, when the absolute neutrophil count (ANC) falls to $<1000$ per $\mu \mathrm{L}$, lenalidomide treatment should be interrupted and treatment with G-CSF initiated with weekly follow-up complete blood counts. When the ANC increases to $\geq 1000$ per $\mu \mathrm{L}$ and neutropenia is the only toxicity, lenalidomide should be resumed at $25 \mathrm{mg}$ /day or at $15 \mathrm{mg} /$ day if there is another toxicity. For each subsequent fall to $<1000$ per $\mu \mathrm{L}$, treatment should again be interrupted and resumed at $5 \mathrm{mg}$ less than the previous dose when the ANC recovers to $\geq 1000$ per $\mu \mathrm{L}$. For other grade 3 or 4 toxicities related to lenalidomide, treatment should be interrupted and restarted at the next lower dose level when the toxicity has resolved to grade 2 or lower. Lenalidomide should not be dosed below $5 \mathrm{mg} /$ day. ${ }^{138}$

\section{Place in therapy}

In patients with relapsed or refractory MM who have received 1-3 prior lines of therapy, lenalidomide in combination with high-dose dexamethasone produces significant prolongation of TTP and OS compared with high-dose dexamethasone alone. ${ }^{2,3}$ In patients with previously untreated MM, lenalidomide in combination with low-dose dexamethasone produces a significant survival advantage compared with lenalidomide plus high-dose dexamethasone. ${ }^{84,90}$ In both the newly diagnosed and relapsed or refractory settings, the addition of lenalidomide to high-dose dexamethasone is associated with a higher rate of grade 3 or 4 myelosuppression, and in the absence of appropriate thromboprophylaxis, a higher rate of VTE events compared with high-dose dexamethasone alone..$^{2,3,43,139}$ However, there is level 2 evidence from the MM-009 study that survival is not affected by occurrence of DVT. ${ }^{126}$

The MM-009 and MM-010 pivotal phase III studies provided level 2 evidence in support of lenalidomide plus dexamethasone in the relapsed or refractory setting. After a median follow-up of 17.1 months post-randomization, median OS in the lenalidomide plus high-dose dexamethasone group was 29.6 months in MM-009 and not yet reached in MM-010. ${ }^{2,3}$ In comparison, median OS in the placebo plus high-dose dexamethasone group was 20.2 months in MM-009 and 20.6 months in MM-010. Thus, the addition of lenalidomide to high-dose dexamethasone in patients who have received 1-3 prior therapies is likely to prolong median survival by approximately nine months. After adjusting for crossover of patients initially assigned to dexamethasone alone into the lenalidomide arm, prolongation of survival is likely to be further enhanced. ${ }^{124}$ Level 2 evidence is available in support of this regimen in patients with one or more than one prior lines of therapy, ${ }^{100}$ in patients with or without previous thalidomide exposure, ${ }^{99}$ in patients with or without prior autologous SCT, ${ }^{137}$ and in patients with mild-to-moderate renal impairment. ${ }^{104}$ Furthermore, there is level 2 evidence in support of this regimen in patients aged $\geq 65$ years and in those aged $<65$ years. ${ }^{136}$

There is level 2 evidence in support of lenalidomide plus dexamethasone in patients with newly diagnosed MM from two phase III studies. ${ }^{83,84,90,91}$ In one study, the investigators did not directly compare the lenalidomide-based regimen with a recognized therapy (ie, dexamethasone alone) ${ }^{84,90}$ Instead, patients in each arm received lenalidomide with either high-dose or low-dose dexamethasone. OS was significantly superior in the low-dose dexamethasone group (one-year OS $96 \%$ vs $88 \%$ in the high-dose dexamethasone group; two-year OS $87 \%$ vs $75 \%$ in the high-dose group). ${ }^{84}$ These data compare favorably with other regimens in this setting, including bortezomib monotherapy (one-year survival 80\%). ${ }^{109}$ In the second study, which compared lenalidomide plus dexamethasone with dexamethasone alone, patient accrual was stopped 
early due to external data affecting the acceptability of the control arm. ${ }^{83}$ The one-year survival data did not favor either treatment arm (93\% for lenalidomide plus dexamethasone vs $91 \%$ for dexamethasone alone). A subgroup analysis suggested the presence of abnormal cytogenetics at baseline was associated with a reduced one-year OS rate compared with no abnormal karyotype (one-year OS $82 \%$ vs 97\%, respectively). High-risk cytogenetic abnormalities (HRCA) did not appear to account for this difference (one-year OS in patients with HRCA $100 \%$ vs $92 \%$ without HRCA); however, sample size limited the statistical power of this study. ${ }^{91}$ Significant differences between the two treatment arms were observed in terms of response rates with lenalidomide plus dexamethasone yielding an ORR of $85.3 \%$ and a $\mathrm{CR}$ rate of $22.1 \%$ compared with $51.3 \%$ and $3.8 \%$, respectively, for dexamethasone alone $(P=0.001){ }^{83}$

During clinical development of lenalidomide, it became apparent that addition of the drug to dexamethasone resulted in a higher rate of VTE events than dexamethasone alone. Although early trial protocols did not include thromboprophylaxis, anticoagulation therapy with aspirin or lowmolecular weight heparin is now recommended. Given that anticoagulation therapy has been inconsistently applied during the lenalidomide clinical development program, it is difficult to assess the impact of anticoagulation therapy on VTE. However, level 4 evidence is available from a systematic review of published literature, abstracts, and package inserts to support the hypothesis that aspirin therapy reduces the incidence of VTE events to $<5 \%$ of patients. ${ }^{132}$ A similar review that captured data for thalidomide as well as lenalidomide suggested that lower rates of VTE may be obtained using low-molecular-weight heparins. ${ }^{131}$ The authors of this review concluded that randomized clinical trials of anticoagulation therapies are needed in order to identify appropriate prophylaxis when MM patients receive either lenalidomide or thalidomide with dexamethasone.

Overall, the current evidence base presented herein suggests that lenalidomide has significantly impacted the treatment of MM, and delivered survival benefits to both patients with newly diagnosed, or relapsed or refractory disease. Although lenalidomide is associated with an increased risk of grade 3 or 4 myelosuppression when combined with dexamethasone, these risks can be mitigated through routine monitoring, dose interruptions, and growth factor support where appropriate. Adequate anticoagulation therapy is needed to minimize the risk of VTE, and in this regard further investigation is necessary to determine optimal treatment. The ability to combine lenalidomide with other agents (eg, bortezomib) is an important feature, and as such lenalidomide, together with bortezomib, thalidomide, and glucocorticoids, can be considered "backbone" agents as part of combination therapy in the treatment of MM. ${ }^{140}$

\section{Acknowledgments}

The authors gratefully acknowledge the assistance of Katherine Redman in preparing this paper and also received editorial support in the preparation of this manuscript, funded in part by Celgene. The authors, however, were fully responsible for content and editorial decisions for this manuscript. The authors disclose the following potential conflicts of interest: Drs Richardson, Ghobrial, and Munshi have participated in advisory boards for Millennium and Celgene; Drs Richardson, Schlossman, Ghobrial, and Munshi have received honoraria from Millennium and Celgene; Dr Anderson has acted as consultant, participated in advisory boards, and received honoraria and grants from Millennium, Celgene, and Novartis; Drs Mitsiades, Laubach, and Teru Hideshima declare no potential conflicts of interest.

\section{References}

1. Celgene Corporation. Revlimid ${ }^{\circledR}$ Prescribing Information. 2009. Accessed April 2009. Available from: http://www.revlimid.com/pdf/ REVLIMID_PI.pdf.

2. Dimopoulos M, Spencer A, Attal M, et al; Multiple Myeloma (010) Study Investigators. Lenalidomide plus dexamethasone for relapsed or refractory multiple myeloma. N Engl J Med. 2007;357: 2123-2132.

3. Weber DM, Chen C, Niesvizky R, et al; Multiple Myeloma (009) Study Investigators. Lenalidomide plus dexamethasone for relapsed multiple myeloma in North America. N Engl J Med. 2007a;357: 2133-2142.

4. Bladé J, Samson D, Reece D, et al. Criteria for evaluating disease response and progression in patients with multiple myeloma treated by high-dose therapy and haemopoietic stem cell transplantation. Myeloma Subcommittee of the EBMT. European Group for Blood and Marrow Transplant. Br J Haematol. 1998;102:1115-1123.

5. Kuehl WM, Bergsagel PL. Multiple myeloma: evolving genetic events and host interactions. Nat Rev Cancer. 2002;2:175-187.

6. Greipp PR, San Miguel J, Durie BG, et al. International staging system for multiple myeloma. J Clin Oncol. 2005;23:3412-3420.

7. [ACS] American Cancer Society). Detailed Guide: Multiple Myeloma. 2008. Accessed April 2008. Available from: http:/www.cancer.org/docroot/CRI/CRI_2_3x.asp?dt=30.

8. Piazza FA, Gurrieri C, Trentin L, Semenzato G. Towards a new age in the treatment of multiple myeloma. Ann Hematol. 2007;86:159-172.

9. Kumar S, Rajkumar SV. Thalidomide and lenalidomide in the treatment of multiple myeloma. Eur J Cancer. 2006;42:1612-1622.

10. Jemal A, Siegel R, Ward E, et al. Cancer statistics, 2008. CA Cancer J Clin. 2008;58:71-96.

11. [NCI] National Cancer Institute. Surveillance Epidemiology and End Results (SEER). 2008. Accessed April 2008. Available from: http://seer. cancer.gov/.at: http://seer.cancer.gov/statfacts/html/mulmy.html.

12. [IMWG] International Myeloma Working Group. Criteria for the classification of monoclonal gammopathies, multiple myeloma and related disorders: a report of the International Myeloma Working Group. Br J Haematol. 2003;121:749-757. 
13. Durie BG. New approaches to treatment for multiple myeloma: durable remission and quality of life as primary goals. Clin Lymphoma Myeloma. 2005;6:181-190.

14. Durie BG, Salmon SE. A clinical staging system for multiple myeloma. Correlation of measured myeloma cell mass with presenting clinical features, response to treatment, and survival. Cancer. 1975;36:842-854.

15. [MMRF] Multiple Myeloma Research Foundation. Multiple myeloma: disease overview. 2008. Accessed April 2008. Available from: http:// www.multiplemyeloma.org/downloads/about_myeloma/Disease_Overview.pdf.

16. Mihou D, Katodritou I, Zervas K. Evaluation of five staging systems in 470 patients with multiple myeloma. Haematologica. 2006;91: 1149-1150.

17. Gertz MA. Relevant prognostic features of multiple myeloma and the new International Staging System. Leuk Lymphoma. 2007;48:458-468

18. Rajkumar SV, Greipp PR. Prognostic factors in multiple myeloma. Hematol Oncol Clin North Am. 1999;13:1295-1314.

19. Turesson I, Abildgaard N, Ahlgren T, et al. Prognostic evaluation in multiple myeloma: an analysis of the impact of new prognostic factors. Br J Haematol. 1999;106:1005-1012.

20. Tricot G, Barlogie B, Jagannath $\mathrm{S}$, et al. Poor prognosis in multiple myeloma is associated only with partial or complete deletions of chromosome 13 or abnormalities involving $11 \mathrm{q}$ and not with other karyotype abnormalities. Blood. 1995;86:4250-4256.

21. Tricot G, Sawyer JR, Jagannath $\mathrm{S}$, et al. Unique role of cytogenetics in the prognosis of patients with myeloma receiving high-dose therapy and autotransplants. J Clin Oncol. 1997;15:2659-2666.

22. Facon T, Avet-Loiseau H, Guillerm G, et al; Intergroupe Francophone du Myélome. Chromosome 13 abnormalities identified by FISH analysis and serum beta2-microglobulin produce a powerful myeloma staging system for patients receiving high-dose therapy. Blood. 2001;97:1566-1571.

23. Fonseca R, Blood E, Rue M, et al. Clinical and biologic implications of recurrent genomic aberrations in myeloma. Blood. 2003;101: 4569-4575.

24. Fonseca R, Barlogie B, Bataille R, et al. Genetics and cytogenetics of multiple myeloma: a workshop report. Cancer Res. 2004;64: 1546-1558.

25. Richardson PG, Barlogie B, Berenson J, et al. A phase 2 study of bortezomib in relapsed, refractory myeloma. $N$ Engl J Med. 2003;348:2609-2617.

26. Durie BG, Harousseau JL, Miguel JS, et al. International Myeloma Working Group International uniform response criteria for multiple myeloma. Leukemia. 2006;20:1467-1473.

27. [NCCN] National Comprehensive Cancer Network ${ }^{\mathbb{R}}$ ). NCCN Clinical Practice Guidelines in Oncology ${ }^{\mathrm{TM}}$. Multiple myeloma. V.2. Accessed August 2009. Available from: http:/www.nccn.org/professionals/ physician_gls/PDF/myeloma.pdf.

28. Anderson KC, Kyle RA, Rajkumar SV, et al; ASH/FDA Panel on Clinical Endpoints in Multiple Myeloma. Clinically relevant end points and new drug approvals for myeloma. Leukemia. 2007;22:231-239.

29. Smith A, Wisloff F, Samson D; UK Myeloma Forum; Nordic Myeloma Study Group; British Committee for Standards in Haematology. Guidelines on the diagnosis and management of multiple myeloma 2005. Br J Haematol. 2006;132:410-451.

30. Bergsagel PL. A kinder, gentler way: control of the proliferative tumor compartment, not cosmetic complete response, should be the goal of myeloma therapy. Leukemia. 2008;22:673-675.

31. Hjorth M, Hellquist L, Holmberg E, Magnusson B, Rödjer S, Westin J. Initial versus deferred melphalan-prednisone therapy for asymptomatic multiple myeloma stage I - a randomized study. Myeloma Group of Western Sweden. Eur J Haematol. 1993;50:95-102.

32. Grignani G, Gobbi PG, Formisano R, et al. A prognostic index for multiple myeloma. Br J Cancer. 1996;73:1101-1107.

33. Kumar S, Lacy MQ, Dispenzieri A, et al. Single agent dexamethasone for pre-stem cell transplant induction therapy for multiple myeloma. Bone Marrow Transplant. 2004;34:485-490.
34. Kumar A, Loughran T, Alsina M, Durie BG, Djulbegovic B. Management of multiple myeloma: a systematic review and critical appraisal of published studies. Lancet Oncol. 2003;4:293-304.

35. Kyle RA, Rajkumar SV. Multiple myeloma. $N$ Engl J Med. 2004;351:1860-1873.

36. Attal M, Harousseau JL, Leyvraz S, et al; Inter-Groupe Francophone du Myélome (IFM). Maintenance therapy with thalidomide improves survival in patients with multiple myeloma. Blood. 2006;108:3289-3294.

37. Mihelic R, Kaufman JL, Lonial S. Maintenance therapy in multiple myeloma. Leukemia. 2007;21:1150-1157.

38. Orlowski RZ. Initial therapy of multiple myeloma patients who are not candidates for stem cell transplantation. Hematology Am Soc Hematol Educ Program. 2006;338-347.

39. Jagannath S. Treatment of myeloma in patients not eligible for transplantation. Curr Treat Options Oncol. 2005;6:241-253.

40. San Miguel JF, Schlag R, Khuageva NK, et al; VISTA Trial Investigators. Bortezomib plus melphalan and prednisone for initial treatment of multiple myeloma. $N$ Engl J Med. 2008;359:906-917.

41. Palumbo A, Falco P, Corradini P, et al; GIMEMA - Italian Multiple Myeloma Network. Melphalan, prednisone, and lenalidomide treatment for newly diagnosed myeloma: a report from the GIMEMA - Italian Multiple Myeloma Network. J Clin Oncol. 2007;25:4459-4465.

42. Rajkumar SV, Hayman SR, Lacy MQ, et al. Combination therapy with lenalidomide plus dexamethasone (Rev/Dex) for newly diagnosed myeloma. Blood. 2005;106:4050-4053.

43. Lacy MQ, Gertz MA, Dispenzieri A, et al. Long-term results of response to therapy, time to progression, and survival with lenalidomide plus dexamethasone in newly diagnosed myeloma. Mayo Clin Proc. 2007;82:1179-1184.

44. Thomas S, Alexanian R. Current treatment strategies for multiple myeloma. Clin Lymphoma Myeloma. 2007;7(Suppl 4):S139-S144.

45. Voorhees PM, Orlowski RZ. Emerging role of novel combinations for induction therapy in multiple myeloma. Clin Lymphoma Myeloma. 2006;7:33-41.

46. [FDA] US Food and Drug Administration. Accessed October 2008. FDA approves lenalidomide oral capsules (Revlimid) for use in combination with dexamethasone in patients with multiple myeloma. 2006. Available from: http://www.fda.gov/cder/Offices/OODP/whatsnew/ lenalidomide.htm.

47. Rajkumar SV, Fonseca R, Dispenzieri A, et al. Thalidomide in the treatment of relapsed multiple myeloma. Mayo Clin Proc. 2000;75:897-901.

48. Lee CK, Barlogie B, Munshi N, et al. DTPACE: an effective, novel combination chemotherapy with thalidomide for previously treated patients with myeloma. J Clin Oncol. 2003;21:2732-2739.

49. Kumar S, Rajkumar SV. Thalidomide and dexamethasone: therapy for multiple myeloma. Expert Rev Anticancer Ther. 2005;5:759-766.

50. Prince HM, Schenkel B, Mileshkin L. An analysis of clinical trials assessing the efficacy and safety of single-agent thalidomide in patients with relapsed or refractory multiple myeloma. Leuk Lymphoma. 2007b;48:46-55.

51. Dimopoulos MA, Zervas K, Kouvatseas G, et al. Thalidomide and dexamethasone combination for refractory multiple myeloma. Ann Oncol. 2001;12:991-995.

52. Schütt P, Ebeling P, Buttkereit U, et al. Thalidomide in combination with dexamethasone for pretreated patients with multiple myeloma: serum level of soluble interleukin-2 receptor as a predictive factor for response rate and for survival. Ann Hematol. 2005;84:594-600.

53. Terpos E, Mihou D, Szydlo R, et al. The combination of intermediate doses of thalidomide with dexamethasone is an effective treatment for patients with refractory/relapsed multiple myeloma and normalizes abnormal bone remodeling, through the reduction of sRANKL/ osteoprotegerin ratio. Leukemia. 2005;19:1969-1976.

54. Kyriakou C, Thomson K, D'Sa S, et al. Low-dose thalidomide in combination with oral weekly cyclophosphamide and pulsed dexamethasone is a well tolerated and effective regimen in patients with relapsed and refractory multiple myeloma. Br J Haematol. 2005;129:763-770. 
55. Prince HM, Adena M, Smith DK, Hertel J. Efficacy of single-agent bortezomib vs single-agent thalidomide in patients with relapsed or refractory multiple myeloma: a systematic comparison. Eur J Haematol. 2007;79:93-99.

56. Richardson PG, Sonneveld P, Schuster MW, et al; Assessment of Proteasome Inhibition for Extending Remissions (APEX) Investigators. Bortezomib or high-dose dexamethasone for relapsed multiple myeloma. N Engl J Med. 2005;106:2547352:2487-2498.

57. Richardson PG, Sonneveld P, Schuster M, et al. Extended follow-up of a phase 3 trial in relapsed multiple myeloma: final time-to-event results of the APEX trial. Blood. 2007;110:3557-3560.

58. Jagannath S, Barlogie B, Berenson J, et al. A phase 2 study of two doses of bortezomib in relapsed or refractory myeloma. Br J Haematol. 2004;127:165-172.

59. Kropff MH, Bisping G, Wenning D, et al. Bortezomib in combination with dexamethasone for relapsed multiple myeloma. Leuk Res. 2005;29:587-590.

60. Popat R, Oakervee H, Williams C, et al. Bortezomib, low dose intravenous melphalan and dexamethasone for patients with relapsed multiple myeloma [abstract]. Haematologica. 2008;93(Suppl 1):0918.

61. Orlowski RZ, Nagler A, Sonneveld P, et al. Randomized phase III study of pegylated liposomal doxorubicin plus bortezomib compared with bortezomib alone in relapsed or refractory multiple myeloma: combination therapy improves time to progression. J Clin Oncol. 2007;25:3892-3901.

62. Richardson P, Jagannath S, Raje N, et al. Lenalidomide, bortezomib, and dexamethasone (Rev/Vel/Dex) in patients with relapsed or relapsed/ refractory multiple myeloma (MM): preliminary results of a phase II study [abstract]. Blood. 2007;110. Abstract 2714.

63. Richardson P, Mitsiades C, Schlossman R, et al. The treatment of relapsed and refractory multiple myeloma. Hematology Am Soc Hematol Educ Program. 2007;317-323.

64. Badros Z, Philip S, Niesvizky R, et al. Phase I trial of vorinostat plus bortezomib (Bort) in relapsed and refractory multiple myeloma (MM) patients (pts) [abstract]. Haematologica. 2008;93(Suppl 1):0642.

65. Weber DM, Jagannath S, Mazumder A, et al. Phase I trial of oral vorinostat in combination with bortezomib in advanced multiple myeloma [abstract]. Haematologica. 2008;93(Suppl 1). Abstract 0640.

66. Palumbo A, Ambrosini MT, Benevolo G, et al; Italian Multiple Myeloma Network; Gruppo Italiano Malattie Ematologicche dell'Adulto. Bortezomib, melphalan, prednisone, and thalidomide for relapsed multiple myeloma. Blood. 2007;109:2767-2772.

67. Zangari M, Anaissie E, Barlogie B, et al. Increased risk of deep-vein thrombosis in patients with multiple myeloma receiving thalidomide and chemotherapy. Blood. 2001;98:1614-1615.

68. Zangari M, Barlogie B, Thertulien R, et al. Thalidomide and deep vein thrombosis in multiple myeloma: risk factors and effect on survival. Clin Lymphoma. 2003;4:32-35.

69. Kastritis E, Dimopoulos MA. The evolving role of lenalidomide in the treatment of hematologic malignancies. Expert Opin Pharmacother. 2007;8:497-509.

70. [EMEA] European Medicines Agency. Scientific Discussion, 2007. Available from: http://www.emea.europa.eu/humandocs/PDFs/EPAR revlimid/H-717-en6.pdf. Accessed October 2008.

71. Anderson KC. Lenalidomide and thalidomide: mechanisms of action - similarities and differences. Semin Hematol. 2005;42(4 Suppl 4): S3-S8.

72. Hideshima T, Mitsiades C, Tonon G, et al. Understanding multiple myeloma pathogenesis in the bone marrow to identify new therapeutic targets. Nat Rev Cancer. 2007;7:585-598.

73. Bartlett JB, Dredge K, Dalgleish AG. The evolution of thalidomide and its IMiD derivatives as anticancer agents. Nat Rev Cancer. 2004;4: 314-322.

74. Hideshima T, Chauhan D, Shima Y, Richardson PG, Anderson KC. Thalidomide and its analogs overcome drug resistance of human multiple myeloma cells to conventional therapy. Blood. 2000;96: 2943-2950.
75. Verhelle D, Corral LG, Wong K, et al. Lenalidomide and CC-4047 inhibit the proliferation of malignant $\mathrm{B}$ cells while expanding normal CD34+ progenitor cells. Cancer Res. 2007;67:746-755.

76. List AF. Lenalidomide - the phoenix rises. $N$ Engl J Med. 2007;357:2183-2186.

77. Bruno B, Rotta M, Giaccone L, et al. New drugs for treatment of multiple myeloma. Lancet Oncol. 2004;5:430-442.

78. Knight R. IMiDs: a novel class of immunomodulators. Semin Oncol. 2005;32(4 Supp1 5):S24-S30.

79. Corral LG, Haslett PA, Muller GW, et al. Differential cytokine modulation and $T$ cell activation by two distinct classes of thalidomide analogues that are potent inhibitors of TNF-alpha. J Immunol. 1999;163:380-386.

80. Mitsiades CS, Mitsiades N, Koutsilieris M. The Akt pathway: molecular targets for anti-cancer drug development. Curr Cancer Drug Targets. 2004; 4:235-256.

81. Dredge K, Horsfall R, Robinson SP, et al. Orally administered lenalidomide (CC-5013) is anti-angiogenic in vivo and inhibits endothelial cell migration and Akt phosphorylation in vitro. Microvasc Res. 2005;69:56-63.

82. Davies FE, Raje N, Hideshima T, et al. Thalidomide and immunomodulatory derivatives augment natural killer cell cytotoxicity in multiple myeloma. Blood. 2001;98:210-216.

83. Zonder JA, Crowley J, Hussein MA, et al. Superiority of lenalidomide (Len) plus high-dose dexamethasone (HD) compared to HD alone as treatment of newly-diagnosed multiple myeloma (NDMM): results of the randomized, double-blinded, placebo-controlled SWOG trial S0232 [abstract]. Blood. 2007;110:77.

84. Rajkumar SV, Jacobus S, Callander N, et al. Randomized trial of lenalidomide plus high-dose dexamethasone versus lenalidomide plus low-dose dexamethasone in newly diagnosed myeloma (E4A03), a trial coordinated by the Eastern Cooperative Oncology Group: Analysis of response, survival, and outcome [abstract]. J Clin Oncol. 2008;26:(Suppl):8504.

85. Kumar S, Hayman SR, Buadi FK, et al. Phase II trial of lenalidomide, cyclophosphamide, and dexamethasone (CRd) for newly diagnosed myeloma [abstract]. Blood. 2007;110:190.

86. Richardson PG, Lonial S, Jakubowiak A, et al. Safety and efficacy of lenalidomide (Len), bortezomib (Bz), and dexamethasone (Dex) in patients (pts) with newly diagnosed multiple myeloma (MM): A phase I/II study [abstract]. J Clin Oncol. 2008;26:(Suppl):8520.

87. Niesvizky R, Jayabalan DS, Christos PJ, et al. BiRD (Biaxin [clarithromycin]/Revlimid [lenalidomide]/dexamethasone) combination therapy results in high complete- and overall-response rates in treatment-naive symptomatic multiple myeloma. Blood. 2008;111:1101-1109.

88. Mark T, Jayabalan D, Pearse RN, et al. Atypical serum immunofixation pattern (ASIP) development during induction therapy with BiRD for newly diagnosed multiple myeloma correlates with a high rate of complete remission [abstract]. Blood. 2007;110:2737.

89. Palumbo AP, Falco T, Corradini P, et al. Bortezomib, pegylated-lyposomaldoxorubicin and dexamethasone (PAD) as induction therapy prior to reduced intensity autologous stem cell transplant (ASCT) followed by lenalidomide and prednisone (LP) as consolidation and lenalidomide alone as maintenance [abstract]. J Clin Oncol. 2008;26(Suppl):8518.

90. Rajkumar SV, Jacobus S, Callander N, et al. A randomized trial of lenalidomide plus high-dose dexamethasone (RD) versus lenalidomide plus low-dose dexamethasone ( $\mathrm{Rd}$ ) in newly diagnosed multiple myeloma (E4A03): a trial coordinated by the Eastern Cooperative Oncology Group [abstract]. Blood. 2007;110:74.

91. Zonder JA, Crowley JJ, Bolejack V, et al. A randomized Southwest Oncology Group study comparing dexamethasone (D) to lenalidomide + dexamethasone (LD) as treatment of newly-diagnosed multiple myeloma (NDMM): Impact of cytogenetic abnormalities on efficacy of LD, and updated overall study results [abstract]. J Clin Oncol. 2008;26:(Suppl):8521.

92. Palumbo A, Falco P, Corradini P, et al. Melphalan, prednisone and lenalidomide for newly diagnosed myeloma: kinetics of neutropenia/ thrombocytopenia and time to event results [abstract]. Haematologica. 2008;93(Suppl 1):0213. 
93. Kumar S, Dispenzieri A, Lacy MQ, et al. Impact of lenalidomide therapy on stem cell mobilization and engraftment post-peripheral blood stem cell transplantation in patients with newly diagnosed myeloma. Leukemia. 2007;21:2035-2042.

94. Paripati H, Stewart AK, Fonseca R, et al. Impact of lenalidomide therapy on stem cell mobilization in myeloma [abstract]. J Clin Oncol. 2008;26(Suppl):8543.

95. Cook RJ, Vogl D, Mangan PA, et al. Lenalidomide and stem cell collection in patients with multiple myeloma [abstract]. J Clin Oncol. 2008;26(Suppl):8547.

96. Mark T, Stern J, Furst J, et al. Stem cell mobilization with cylophosphamide overcomes the suppressive effect of lenalidomide therapy on stem cell collection in multiple myeloma. Biol Blood Marrow Transplant. 2008;14:795-798.

97. Weber D, Knight R, Chen C, et al. Prolonged overall survival with lenalidomide plus dexamethasone compared with dexamethasone alone in patients with relapsed or refractory multiple myeloma [abstract]. Blood. 2007;110:412.

98. Harousseau J-L, Weber D, Dimopoulos M, et al. Relapsed/refractory multiple myeloma patients treated with lenalidomide/dexamethasone who achieve a complete or near complete response have longer overall survival and time to progression compared with patients achieving a partial response [abstract]. Blood. 2007;110:3598.

99. Wang M, Knight R, Dimopoulos D, et al. Effect of Len/Dex in MM despite Thal resistance [abstract]. Haematol Hematol J Haematologica. 2007;92(s2):6 Suppl 2):PO-662.

100. Stadtmauer E, Weber D, Dimopoulos M, et al. Lenalidomide in combination with dexamethasone is more effective than dexamethasone at first relapse in relapsed multiple myeloma [abstract]. Blood. 2006;108:3552.

101. Foa R, Weber D, Dimopoulos M, et al. Lenalidomide/dexamethasone improves response and prolongs time to progression, even in patients with IgA multiple myeloma: a sub-analysis of the MM-009/010 studies [abstract]. Blood. 2007;110:4839.

102. Chanan-Khan AA, Dimopoulos M, Weber D, et al. ECOG performance status affects efficacy, but not safety, of lenalidomide/dexamethasone in relapsed/refractory multiple myeloma (MM009/010 sub-analysis) [abstract]. Blood. 2007;110:2721.

103. Lonial S, Knight R, Dimopoulos M, et al. Effect of Len/Dex in MM in different age groups. Haematologica. 2007;92(6 Suppl 2):PO-663.

104. Weber DM, Spencer A, Wang M, et al; MM-009 and MM-010 Investigators. The efficacy and safety of lenalidomide plus dexamethasone in relapsed or refractory multiple myeloma patients with impaired renal function [abstract]. J Clin Oncol. 2008;26(Suppl):8542.

105. San Miguel JF, Dimopoulos M, Weber D, et al. Dexamethasone dose adjustments seem to result in better efficacy and improved tolerability in patients with relapsed/refractory multiple myeloma who are treated with lenalidomide/dexamethasone (MM-009/010 sub-analysis) [abstract]. Blood. 2007;110:2712.

106. Kneppers E, Lokhorst HM, Eeltink C, et al. Pretreatment with thalidomide and bortezomib does not influence response to lenalidomide in relapsed myeloma. Preliminary results from patients included in Dutch compassionate need programme [abstract]. Haematologica. 2008;93(Suppl 1):0651.

107. Richardson PG, Blood E, Mitsiades CS, et al. A randomized phase 2 study of lenalidomide therapy for patients with relapsed or relapsed and refractory multiple myeloma. Blood. 2006;108:3458-3464.

108. Jagannath S, Richardson PG, Zeldenrust S, et al. Long-term responses observed with lenalidomide therapy for patients with relapsed or refractory multiple myeloma [abstract]. J Clin Oncol. 2008;26(Suppl):8525.

109. Richardson P, Sonneveld P, Schuster M, et al. Bortezomib continues to demonstrate superior efficacy compared with high-dose dexamethasone in relapsed multiple myeloma: updated results of the APEX trial [abstract]. Blood. 2005;106:2547.

110. Richardson PG, Jagannath S, Avigan DE, et al. Lenalidomide plus bortezomib (Rev-Vel) in relapsed and/or refractory multiple myeloma (MM): final results of a multicenter phase 1 trial [abstract]. Blood. 2006;108:405.
111. Richardson P, Jagannanth S, Hussein M, et al. Safety and efficacy of single-agent lenalidomide in patients with relapsed and refractory multiple myeloma. Blood. 2009;114:772-778.

112. Hussein MA, Richardson PG, Jagannath S, et al. Final analysis of MM-014: single-agent lenalidomide in patients with relapsed and refractory multiple myeloma [abstract]. J Clin Oncol. 2008; 26(Suppl):8524.

113. Richardson PG, Schlossman RL, Weller E, et al. Immunomodulatory drug CC-5013 overcomes drug resistance and is well tolerated in patients with relapsed multiple myeloma. Blood. 2002;100: 3063-3067.

114. Baz R, Walker E, Karam MA, et al. Lenalidomide and pegylated liposomal doxorubicin-based chemotherapy for relapsed or refractory multiple myeloma: safety and efficacy. Ann Oncol. 2006;17: 1766-1771.

115. Morgan GJ, Schey SA, Wu P, et al. Lenalidomide (Revlimid), in combination with cyclophosphamide and dexamethasone (RCD), is an effective and tolerated regimen for myeloma patients. Br J Haematol. 2007;137:268-269.

116. Knop S, Gerecke C, Liebisch P, et al. The efficacy and toxicity of the RAD regimen (Revlimid $^{\circledR}$, Adriamycin ${ }^{\circledR}$, dexamethasone) in relapsed and refractory multiple myeloma - a phase I/II trial of "Deutsche Studiengruppe Multiples Myelom" [abstract]. Blood. 2007;110:2716.

117. Knop S, Gerecke C, Liebisch P, et al. Results of a phase I/II trial of Deutsche Studiengruppe Multiples Myelom, showing efficacy and safety of RAD regimen (Revlimid ${ }^{\circledR}$, Adriamycin ${ }^{\circledR}$, dexamethasone) in relapsed/refractory multiple myeloma [abstract]. Haematologica. 2008;93(Suppl 1):0637.

118. Reece DE, Masih-Khan E, Chen C, et al. Lenalidomide (Revlimid ${ }^{\circledR}$ ) $+/-$ corticosteroids in elderly patients with relapsed/refractory multiple myeloma [abstract]. Blood. 2006;108:1013a-1014a, \#. 3550.

119. Anderson KC, Jagannath S, Jakubowiak A, et al. Phase II study of lenalidomide (Len), bortezomib (Bz), and dexamethasone (Dex) in patients (pts) with relapsed or relapsed and refractory multiple myeloma (MM) [abstract]. J Clin Oncol. 2008;26(Suppl):8545.

120. Terpos E, Christoulas D, Kastritis E, et al. Effect of lenalidomidebased regimens on bone remodeling in patients with relapsed/ refractory multiple myeloma [abstract]. Haematologica. 2008; 93(Suppl 1):0192.

121. Raschko M, Markovina S, Miyamoto S, et al. Phase II trial of bevacizumab combined with low dose dexamethasone and lenalidomide (Bev/Rev/Dex) for relapsed or refractory myeloma (MM) [abstract]. Blood. 2007;110:1173.

122. Palumbo A, Falco P, Sanpaolo G, et al. Lenalidomide, melphalan, prednisone and thalidomide (RMPT) for relapsed/refractory multiple myeloma [abstract]. Haematologica. 2008;93(Suppl 1):0636.

123. Stadtmauer EA, Lonial S, Vesole DH, et al. A practical guide to achieving and maintaining the best response to lenalidomide in multiple myeloma: roundtable proceedings. Clin Adv Hematol Oncol. 2007;5(10 Suppl 15):7-19.

124. Morgan J, Ishak K, Deniz B, et al. Overall survival with dexamethasone in phase III multiple myeloma trials after adjustment for cross-over to lenalidomide [abstract]. Haematologica. 2008; 93(Suppl 1):0441.

125. Bahlis NJ, Song K, Trieu Y, et al. Lenalidomide overcomes poor prognosis conferred by del13q and $\mathrm{t}(4 ; 14)$ but not del17p13 in multiple myeloma: results of the Canadian MM016 trial [abstract]. Blood. 2007;110:3597.

126. Zangari M, Fink L, Knight R, et al. Effect on survival of lenalidomide and dexamethasone associated deep vein thrombosis (DVT) in relapsed multiple myeloma patients [abstract]. Haematologica. 2008;93(Suppl 1):0638.

127. Niesvizky R, Spencer A, Wang M, et al. Increased risk of thrombosis with lenalidomide in combination with dexamethasone and erythropoietin [abstract]. J Clin Oncol. 2006; 24:(Suppl):7506. 
128. Chen C, Reece DE, Siegel D, et al. Expanded Access Program (EAP) for lenalidomide $\left(\right.$ Revlimid $^{\circledR}$ ) plus dexamethasone in over $1400 \mathrm{sub}$ jects with relapsed or refractory multiple myeloma [abstract]. Blood. 2006;108:3556.

129. Niesvizky R, Naib T, Christos PJ, et al. Lenalidomide-induced myelosuppression is associated with renal dysfunction: adverse events evaluation of treatment-naïve patients undergoing front-line lenalidomide and dexamethasone therapy. Br J Haematol. 2007;138:640-643.

130. Simpson L, Rajkumar SV, Dispenzieri A, et al. High incidence of diarrhea in patients on long term therapy with lenalidomide and dexamethasone for multiple myeloma [abstract]. J Clin Oncol. 2008;26(Suppl):8586.

131. Bennett CL, Hussain Z, Courtney M, Yarnold P, Raisch D, McKoy JM. RADAR update on thalidomide (Thal)- and lenalidomide (Len)associated venous thromboembolism (VTE): safety concerns persist for multiple myeloma (MM) despite FDA approvals in this setting [abstract]. Blood. 2006;108:3310.

132. Angelotta C, Lurie AJ, Yarnold PR, et al. Black box warning on lenalidomide-associated venous thromboembolism (VTE) in off-label setting: A preemptive and unusual safety initiative [abstract]. J Clin Oncol. 2006;24:(Suppl):6074.

133. Fullerton DSP, Trautman H, Huang H, et al. A budget impact model comparing resource utilization of four approved therapies for multiple myeloma (MM) in the US [abstract]. Blood. 2007;110:3324

134. Armoiry X, Fagnani F, Benboubker L, et al. Estimating direct costs of care for patients with relapsed or refractory multiple myeloma in French hospitals [abstract]. J Clin Oncol. 2008;26(Suppl):8596.
135. Deniz HB, Ishak KJ, Edwards DR, Shearer A, Dale P, Caro JJ Economic evaluation of lenalidomide for the treatment of multiple myeloma in Wales in patients who have received at least one prior therapy [abstract]. Haematologica. 2008;93(Suppl 1):0804.

136. Chanan-Khan AA, Weber D, Dimopoulos M, et al. Lenalidomide (L) in combination with dexamethasone (D) improves survival and time to progression in elderly patients (pts) with relapsed or refractory (rel/ref) multiple myeloma (MM) [abstract]. Blood. 2006a;108:3551.

137. Chanan-Khan AA, Yu Z, Weber D, et al. Lenalidomide (L) in combination with dexamethasone (D) significantly improves time to progression (TTP) in non-stem cell transplant patients (pts) with relapsed or refractory (rel/ref) multiple myeloma (MM): analysis from MM-009 and MM-010 randomized phase III clinical trials [abstract]. Blood. 2006;108:3554

138. Anon. Revlimid (lenalidomide) Prescribing information. 2008. Available from: http://www.revlimid.com/pdf/REVLIMID_PI.pdf. Accessed October 2, 2008.

139. Zonder JA, Durie BG, McCoy J, et al. High incidence of thrombotic events observed in patients receiving lenalidomide $(\mathrm{L})+$ dexamethasone (D) (LD) as first-line therapy for multiple myeloma (MM) without aspirin (ASA) prophylaxis [abstract]. Blood. 2005;106:3455.

140. Richardson RG, Mitsiades C, Schlossman R, et al. New drugs for myeloma. Oncologist. 2007;12:664.

\section{Core Evidence}

\section{Publish your work in this journal}

Core Evidence is an international, peer-reviewed open-access journal evaluating the evidence underlying the potential place in therapy of drugs throughout their development lifecycle from preclinical to postlaunch. The focus of each review is to evaluate the case for a new drug or class in outcome terms in specific indications and patient

\section{Dovepress}

groups. The manuscript management system is completely online and includes a very quick and fair peer-review system, which is all easy to use. Visit http://www.dovepress.com/testimonials.php to read real quotes from published authors. 